\title{
Towards a Unifying Basis of Auditory Thresholds: The Effects of Hearing Loss on Temporal Integration Reconsidered
}

\author{
Heinrich Neubauer and Peter Heil \\ Leibniz Institute for Neurobiology, Magdeburg, Germany
}

Received: 10 May 2004; Accepted: 29 July 2004; Online publication: 18 October 2004

\begin{abstract}
For signal detection and identification, the auditory system needs to integrate sound over time. It is frequently assumed that the quantity ultimately integrated is sound intensity and that the integrator is located centrally. However, we have recently shown that absolute thresholds are much better specified as the temporal integral of the pressure envelope than of intensity, and we proposed that the integrator resides in the auditory pathway's first synapse. We also suggested a physiologically plausible mechanism for its operation, which was ultimately derived from the specific rate of temporal integration, i.e., the decrease of threshold sound pressure levels with increasing duration. In listeners with sensorineural hearing losses, that rate seems reduced, but it is not fully understood why. Here we propose that in such listeners there may be an elevation in the baseline above which sound pressure is effective in driving the system, in addition to a reduction in sensitivity. We test this simple model using thresholds of cats to stimuli of differently shaped temporal envelopes and durations obtained before and after hearing loss. We show that thresholds, specified as the temporal integral of the effective pressure envelope, i.e., the envelope of the pressure exceeding the elevated baseline, behave almost exactly as the lower thresholds, specified as the temporal integral of the total pressure envelope before hearing loss. Thus, the mechanism of temporal integration is likely un-
\end{abstract}

Correspondence to: Peter Heil - Leibniz Insitute of Neurobiology • Brenneckestr. 6 - D-39118 Magedeburg, Germany. Telephone: + 49 391 6263-332; Fax + 49391 6263-328; email: peter.heil@ifn-magdeburg.de changed after hearing loss, but the effective portion of the stimulus is. Our model constitutes a successful alternative to the model currently favored to account for altered temporal integration in listeners with sensorineural hearing losses, viz., reduced peripheral compression. Our model does not seem to be at variance with physiological observations and it also qualitatively accounts for a number of phenomena observed in such listeners with suprathreshold stimuli.

Keywords: temporal summation, cochlear impairment, hearing loss, absolute thresholds, reaction times, recruitment, auditory modeling

\section{INTRODUCTION}

In every species examined, the sound pressure level (SPL) needed for a sound to be detected decreases as sound duration increases (see, e.g., Fay 1992 for review). This trading relationship, sometimes called temporal integration function, is frequently interpreted to indicate that the auditory system integrates sound over time. Until recently, the prevailing view has been that the sound quantity ultimately integrated by the auditory system is sound intensity and that consequently absolute thresholds are best defined in terms of acoustic energy density (Garner 1947; Feldtkeller and Oetinger 1956; Plomp and Bouman 1959; Zwislocki 1960; Dallos and Johnson 1966; Watson and Gengel 1969; Green 1985; Gerken et al. 1990; Schmidt and Thaller 1994; Clock Eddins and Peterson 1999; O'Connor et al. 1999). 
However, there has been no consensus as to where the integrator may be located nor as to how it may operate. For example, it is unclear whether an integration process in the literal or physical sense exists at all and whether it is a deterministic or rather a probabilistic process. Two phenomenological "models", viz., power law and a leaky integrator of intensity, have often been applied to describe the temporal integration functions (for review see Gerken et al. 1990). However, no physiologically plausible explanation for the power law has been offered (see Florentine et al. 1988), and the leaky integrator approach has encountered serious problems because time constants up to several hundreds of milliseconds are needed to fit the data (for summaries see Gerken et al. 1990; O'Connor et al. 1999). Such long time constants contrast with the short membrane time constants of neurons in the auditory periphery and are also difficult to reconcile with the high temporal resolution of the auditory system, creating the "resolution-integration paradox" (deBoer 1985). As a way out, some authors have conjectured that temporal resolution is mediated by peripheral processing and long-term temporal integration (of neural activity directly linked to stimulus intensity) is mediated by processing high up in the central auditory system (Zwislocki 1960; Watson and Gengel 1969; Gerken et al. 1990). Viemeister and Wakefield (1991) proposed that the system only acts as if it were a long-term integrator but instead is a short-term integrator (of intensity) that combines information from multiple, independent, brief samples during the stimulus ("multiple looks"). Dau et al. (1996a,b) have suggested that the system performs a running cross-correlation of the activity pattern triggered by a particular threshold stimulus with a stored template of a pattern produced by some suprathreshold version of that stimulus. However, clear parallels of the perceptual trading relationship between the SPL of a threshold stimulus and its duration have been observed in neuronal responses at very peripheral levels of the auditory system, viz., cochlear nucleus (Kitzes et al. 1978; Clock Eddins et al. 1993) and auditory nerve (Dunia and Narins 1989; Viemeister et al. 1992; Clock Eddins et al. 1998), suggesting that integration is accomplished at, or peripheral to, the auditory nerve.

We have recently shown that absolute thresholds, at both the neuronal and perceptual levels, are phenomenologically much better specified as the temporal integral of the sound's pressure envelope than of its intensity (Heil and Neubauer 2001, 2003, $2004 a, b)$. Our approach was to use stimuli of different durations but also of different envelopes and to search for a specification of threshold which would yield thresholds that are independent of envelope shape. We found this to be nearly so for the temporal integral of the pressure envelope but clearly not for that of intensity. We have also traced the location of the integrator to the first synapse in the auditory pathway and provided a physiologically plausible explanation for its operation, namely, a summation process of chance (possibly exocytotic) events whose rate is pressure-dependent. This probabilistic rather than caloric model was consistent with the typical magnitude of the slope of the temporal integration function.

Sensorineural hearing losses, whether due to noise trauma, presbyacusis, ototoxic drugs, or diseases, are not only characterized by elevated thresholds but also often by significantly shallower slopes of temporal integration functions compared to normal-hearing subjects (e.g., Miskolczy-Fodor 1953; Harris et al. 1958; Henderson 1969; Martin and Wofford 1970; Gengel and Watson 1971; Stelmachowicz and Seewald 1977; Chung and Smith 1980; Dempsey and Maxon 1982; Wall and Stephanson 1982; Davis and Ferraro 1983; Hall and Fernandez 1983; Gorga et al. 1984; Hall and Wood 1984; Kidd et al. 1984; Clark and Bohne 1986; Florentine et al. 1988; Papsin and Abel 1988; Carlyon et al. 1990; Saunders et al. 1995; Oxenham et al. 1997; Buus et al. 1999; Hicks and Bacon 1999; Quaranta et al. 2001; for review see, e.g., Moore and Oxenham 1998). It is not fully understood what causes the altered slopes of temporal integration functions, knowledge that is crucial when attempting to alleviate the problems associated with sensorineural hearing losses. The standard model assumes that the altered slopes result from loss of basilar membrane compression (Moore 1991, 1996), although there is no experimental evidence for such compression at the low SPLs required for absolute thresholds.

Here, we reanalyze perceptual thresholds from cats with sensorineural hearing losses to further constrain our model of temporal summation, and we propose a new and simple explanation for the shallower slopes of temporal integration functions in subjects with sensorineural hearing losses. We use data of Solecki and Gerken (1990) who measured thresholds of the same cats prior to and after induction of a sensorineural hearing loss using identical procedures, so that the effects of the trauma can be quantified in each individual. Solecki and Gerken (1990) showed that the slopes of the temporal integration functions were less steep after hearing loss and concluded that such functions for cats and humans are similar. Most importantly for us, they measured thresholds to an extensive set of stimuli with differently shaped temporal envelopes, which allowed us to apply our previous analytical approach (Heil and Neubauer 2003, 2004a, 2004b) and to see consequences of the hear- 
ing losses, which could not be discovered otherwise. Our analysis, which provides an excellent description of these data, suggests that the slope of the temporal integration function is only seemingly changed by the trauma but in reality is not. Hence, the mechanisms determining this slope are also unchanged in listeners with sensorineural hearing losses. Remarkably, our simple model also accounts for a wide variety of other effects of hearing loss.

\section{METHODS}

Absolute thresholds (in dB SPL) for 5 female adult cats without signs of middle ear disease were kindly given to us by G.M. Gerken and J.M. Solecki. They were measured as detailed in their paper (Solecki and Gerken 1990). Therefore, these methods are only briefly repeated here.

\section{Stimuli}

Thresholds were obtained to sets of single- and multiple-burst stimuli differing in duration and envelope characteristics as illustrated in Figure (Fig. 1a). The carrier frequency was $6.25 \mathrm{kHz}$. All tones were gated with cosine-squared onsets and offsets. The shortest stimulus (stimulus 1) consisted only of onset and offset portions of $4.16 \mathrm{~ms}$ each (corresponding to 26 cycles), without plateau. Stimuli of different durations were generated from stimulus 1 in two different ways. Multiple-burst stimuli (stimuli 2-5) were composed of $2,4,8$, or 16 copies of stimulus 1 with a constant interburst interval of $4.16 \mathrm{~ms}$ (defined as the time from the offset of the preceding to the onset of the succeeding burst). Together with stimulus 1 , these stimuli form the multiple-burst series (stimuli 1-5). A second duration series was created by inserting constant-amplitude plateaus in the middle of stimulus 1 with plateau durations of $8.32,33.28$, $66.56,133.12$, and $266.24 \mathrm{~ms}$ (stimuli 6-10). Together with stimulus 1 , these stimuli form the singleburst series (stimuli 1, 6-10).

\section{Procedure}

Threshold measurements were made with the cats in a soundproof booth, using a paw-withdrawal paradigm and an adaptive procedure. Starting sound level was decreased $10 \mathrm{~dB}$ per hit until the first miss, then increased by $5 \mathrm{~dB}$ until the next hit, and thereafter the level was decreased by $2.5 \mathrm{~dB}$ per hit and increased by $2.5 \mathrm{~dB}$ per miss until 6 reversals had occurred. A threshold was calculated as the average level from the reversals obtained with the 2.5-dB steps and accepted only if the standard deviation (SD)

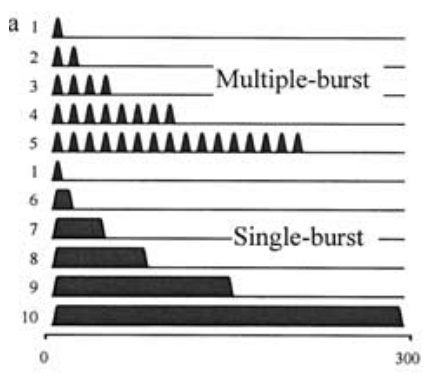

Time (ms)

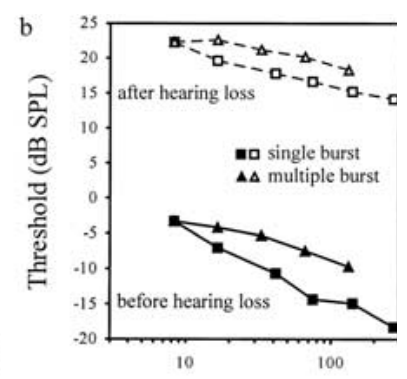

Stimulus duration (ms)
FIG. 1. a. Envelopes of the stimuli for which absolute thresholds of cats were obtained by Solecki and Gerken (1990). Stimuli are assigned to a multiple- (stimuli $1-5$, triangles) and a single-burst series (stimuli, 1, 6-10, squares). b. Grand mean thresholds (in dB SPL; corresponding to the maximum amplitude of each stimulus), obtained from the cats before (filled symbols) and after (open symbols) hearing loss and plotted against physical stimulus duration.

within the data set for that threshold was $\leq 4 \mathrm{~dB}$ and if the number of anticipatory responses within $10 \mathrm{~dB}$ of threshold did not exceed the number of hits. Each cat was tested until 12 acceptable thresholds for each of the 10 stimuli were obtained. Each individual threshold reported here is the mean of 12 such accepted thresholds for a given stimulus and cat.

To induce hearing loss, each cat was exposed for $48 \mathrm{~h}$ to a continuous $2-\mathrm{kHz}$ tone of $110 \mathrm{~dB}$ SPL in an isolated exposure room. Cats were not anesthetized during the exposure. Postexposure temporal integration testing started no sooner than 27 days after the exposure was terminated to allow for recovery and retraining. A second set of 12 acceptable thresholds for each of the 10 stimuli was then collected from each cat.

\section{Specifications of thresholds}

In this study, thresholds are specified in different ways. One specification, which is that also most routinely used in the literature, is in terms of $\mathrm{dB}$ SPL, i.e., of the sound pressure level in $\mathrm{dB}$ re $20 \mu \mathrm{Pa}$ corresponding to the stimulus peak amplitude. Thresholds are also specified in terms of the pressure envelope $P(t)$ integrated over the stimulus duration, or in terms of the intensity $I(t)$ integrated over the stimulus duration, i.e., of energy density. The latter is proportional to $P^{2}(t)$ integrated over the stimulus duration, since for pure tones $I(t)$ is proportional to $P^{2}(t)$ (Buser and Imbert 1992). The use of these integral measures of thresholds shall not imply that the auditory system does reach threshold by an integration process in the literal or physical sense. In other words, we do not assume the existence of a response which is directly proportional to the continuously increasing integral of $P(t)$ or of $P(t)$ raised to some power and which could be measured during a given trial. We rather assume that temporal 
integration is a statistical summation process (Heil and Neubauer 2003). On this view, threshold corresponds to a (possibly statistically distributed) number of individual events (e.g., exocytotic events at the inner hair cell to AN fiber synapses or events of coincidence of spikes at higher levels of the system) which is reached after a statistically distributed amount of time. Thus, the relationship between the integral measures of thresholds and stimulus detection is not deterministic but probabilistic.

\section{Definition of stimulus duration}

Unlike previous studies on temporal integration by other authors but consistent with ours on this topic (Heil and Neubauer 2003, 2004a,b), we initially define stimulus duration as the total duration during which sound is present (henceforth referred to as physical duration). This definition thus excludes all silent intervals and it does not weight the durations of onsets and offsets any different than the duration of a plateau. Of course, the stimulus amplitudes during the onset and offset times are lower, and consequently any integrated measure grows more slowly during such times than during the plateau. Later, and explained in the Results, we also introduce the concept of an effective stimulus duration.

\section{Data analysis}

We obtained grand mean thresholds by first averaging the threshold SPLs obtained from each cat for a given stimulus. Grand mean thresholds specified in other ways were then calculated from these grand mean threshold SPLs and the appropriate stimulus specifications. For thresholds specified in terms of integrated pressure envelope $P(t)$, for example, this is equivalent to calculating the geometric mean of individual thresholds specified in Pa s.

We found (Heil and Neubauer 2003) that thresholds to tones of the same physical duration but of different temporal envelopes were rather similar when specified in terms of the temporal integral of $P(t)$ but not when specified in terms of the temporal integral of intensity $I(t)$. Here, we quantify the similarity of thresholds to stimuli of the single- and the multiple-burst series of corresponding physical duration, allowing us to critically assess our model. For a given physical stimulus duration, we first calculated a coefficient of variation $C V$ :

$$
C V=\sqrt{\left(T_{\mathrm{SB}}-T_{\mathrm{M}}\right)^{2}+\left(T_{\mathrm{MB}}-T_{\mathrm{M}}\right)^{2}} / T_{\mathrm{M}}
$$

where $T_{\mathrm{SB}}$ and $T_{\mathrm{MB}}$ are the thresholds to a single- and a multiple-burst stimulus of the same physical dura- tion, and $T_{\mathrm{M}}$ is their mean. Threshold itself is defined as

$$
T\left(t_{\mathrm{s}}\right)=\int_{0}^{t_{\mathrm{s}}} P(t)^{q} d t
$$

where $t_{\mathrm{s}}$ is the physical stimulus duration and the exponent $q$ is the parameter on which $C V$ depends. The measure of $C V$ thus normalizes the variation in thresholds by the mean threshold at every stimulus duration. A measure without such a normalization would be dominated by large absolute variations. They occur when thresholds, as just defined, are large, i.e., for long stimulus durations. The coefficients were then subjected to a least-squares method for estimation of $P_{\text {ineff. }}$ For this purpose, the coefficients were squared and averaged across all points to yield the mean $(C V)^{2}$. This measure is the smaller, the larger the overall similarity of thresholds to stimuli of the single- and the multiple-burst series, and is therefore referred to as a measure of difference. Its square root, multiplied by 100 , yields a mean $C V$ in percent. Since $C V$ operates on the ratio level, it requires thresholds in linear values (e.g., in $\mu \mathrm{Pa} \mathrm{s}$ if $q=1$ ), but not in logarithmic values (e.g., in $\mathrm{dB}$ ). We first generated 1000 supporting points, equally spaced over a logarithmic axis of duration from 1 to $275 \mathrm{~ms}$. Thresholds at those supporting points were obtained via linear interpolation on double-log axes where this was possible. $C V$ was calculated for all durations for which two interpolated thresholds, i.e., one from each stimulus series, were available. Before hearing loss, this was the case for 493 supporting points ranging from about 8.32 to $133.12 \mathrm{~ms}$.

\section{RESULTS}

\section{Thresholds before hearing loss}

The grand mean thresholds before hearing loss, specified in terms of dB SPL, decrease with increasing physical stimulus duration, irrespective of whether that duration is altered by changing the number of bursts (multiple-burst series) (Fig. 1b, filled triangles) or the plateau duration of a single burst (singleburst series) (Fig. 1b, filled squares). However, the slope is steeper for the single- than for the multipleburst stimuli, so that threshold SPLs for the two types of stimuli differ systematically. Thresholds, specified in terms of the temporal integral of $P(t)$ (in $\mu \mathrm{Pa} \mathrm{s}$ ), increase with increasing physical duration, but, importantly, thresholds for single- and multiple-burst stimuli of any given duration are very similar. There are no obvious systematic differences between them (Fig. 2a,b, filled symbols; panels c,d are described later). This similarity constituted our main argument 
that thresholds are better specified as the temporal integral of $P^{1}(t)$ than of $P^{2}(t)$ or of $I(t)$, because with the latter measure there are obvious systematic differences (Heil and Neubauer 2003; see also Fig. 8a,b). The increase of thresholds, specified in terms of integrated $P(t)$, with physical stimulus duration (Fig. 2a,b, filled symbols) is well-described by the power law:

$$
T\left(t_{\mathrm{s}}\right)=\int_{0}^{t_{\mathrm{s}}} P(t) d t=k \cdot t_{\mathrm{s}}^{m}
$$

Parameter $k$ is an inverse measure of sensitivity and defines the curve's vertical position, while $m$ corresponds to the slope of the increase in threshold $T\left(t_{\mathrm{s}}\right)$ with physical stimulus duration $t_{\mathrm{s}}$ in double-log scales. The slight downward curvature in these data may be coincidental or result from some unknown aspect of the experimental methods used to obtain the thresholds. Such a curvature was neither seen in data from the same species, obtained from a study by Costalupes (1983), nor in data from other species, which were also all well-described by Eq. (1) (see Fig. 3d in Heil and Neubauer 2003). Fits of Eq. (1) to the grand mean data yielded a value of $m=0.69$, and fits to the data from the individual cats yielded values which range from 0.60 to 0.76 . The same fits yielded extrapolated thresholds $T_{1}$ (in $\mu \mathrm{Pa}$ s), i.e., $T\left(t_{\mathrm{s}}\right)$ for $t_{\mathrm{s}}=1 \mathrm{~ms}$, of $0.021 \mu \mathrm{Pa}$ s for the grand mean data and values which range from 0.008 to $0.061 \mu \mathrm{Pa} \mathrm{s}$ in individuals. $T_{1}$ has the same numerical value as $k$ when $k$ is expressed in $\mu \mathrm{Pa} /\left(\mathrm{ms}^{m}\right)$. The smaller $T_{1}$, is the higher is the sensitivity.

\section{Thresholds after hearing loss}

Figure $1 \mathrm{~b}$ also shows the grand mean threshold SPLs of the same cats obtained after induction of sensorineural hearing losses. Compared to before the hearing losses, threshold SPLs are elevated by about 25-32 $\mathrm{dB}$, depending on the stimulus. A reduced slope of the temporal integration function after hearing loss is also evident from Figure 1b, most clearly for the single-burst stimuli. In Figure 2a, the same thresholds are plotted but now specified in terms of integrated $P(t)$. These thresholds are elevated by more than an order of magnitude and the slope of the increase in thresholds with physical stimulus duration is steeper after than before hearing loss. The elevation is captured by larger values of $T_{1}$ (in $\mu \mathrm{Pa}$ s) and the steeper increase by a larger power $m$. Fits of Eq. (1) to the grand mean data (single- and multiple-burst stimuli combined) yielded a value of $m=0.89$, and fits to the data from the individual cats yielded values which range from 0.80 to 0.95 . In Figure $2 b$, the grand mean thresholds of Figure 2a are replotted but now shifted relative to each other (by a factor of 19.1) such that the thresholds before and after hearing loss for the shortest stimulus (of 8.32-ms physical duration) are superimposed. The overall loss of sensitivity due to the trauma can still be seen by comparing the numbers on the left and right ordinates, which identify the thresholds before and after hearing loss, respectively. The advantage of this shift is a better resolution and appreciation of the steeper increase in the thresholds with physical stimulus duration after hearing loss. The plot also shows more clearly that after hearing loss the thresholds for multiple- and single-burst stimuli no longer match. This effect was not detected by Solecki and Gerken (1990) since they specified thresholds in dB SPL. The increase of thresholds for stimuli of the single-burst series with physical stimulus duration is considerably steeper, and consequently thresholds are systematically higher than those for stimuli of the multiple-burst series (Fig. 2a,b).

\section{The model: noise trauma leads to changes in gain and baseline}

These effects of hearing loss lead to the idea that the noise trauma may have had two different consequences. One is a reduction in sensitivity, captured by parameter $k$ in Eq. (1). This is equivalent to a simple change in gain, possibly, but not necessarily, due to a change in the active mechanism associated with outer hair cells. The other consequence may be a shift of the baseline above which the sound pressure can be effective at all. In other words, after the trauma there may be some (elevated) limit, $P_{\text {ineff }}$ (in $\mathrm{Pa}$ ), below which the sound pressure is entirely ineffective in driving the system, and only that part of $P(t)$ which exceeds $P_{\text {ineff, viz., }} P_{\text {eff }}(t)$, can affect the system. Apart from those two effects, the model assumes that temporal summation remains unchanged. We refer to this model as the elevated baseline temporal summation (EBTS) model. After hearing loss, thresholds, specified in terms of integrated $P_{\text {eff }}(t)$, should then increase with effective stimulus duration $t_{\text {eff }}$ (see below) according to a power law much like Eq. (1):

$$
T\left(t_{\mathrm{eff}}\right)=\int_{0}^{t_{\mathrm{eff}}} P_{\mathrm{eff}}(t) d t=k \cdot t_{\mathrm{eff}}^{m}
$$

where $P_{\text {eff }}(t)=\left[P(t)-P_{\text {ineff }}\right]$, when $P(t)>P_{\text {ineff. }}$ $P_{\text {eff }}(t)=0$, when $P(t) \leq P_{\text {ineff. The first instant in }}$ time at which $P(t)=P_{\text {ineff }}$ marks $t=0$. Equation (1) constitutes the special case of Eq. (2) when $P_{\text {ineff }}=0$ and hence $P_{\text {eff }}(t)=P(t)$, which describes the normalhearing situation very well (Heil and Neubauer 2003). This is not to say that in normal-hearing sub- 


$$
T\left(t_{s}\right)=\int_{0}^{t_{s}} P^{1}(t) d t
$$

a

$\mathrm{b}$
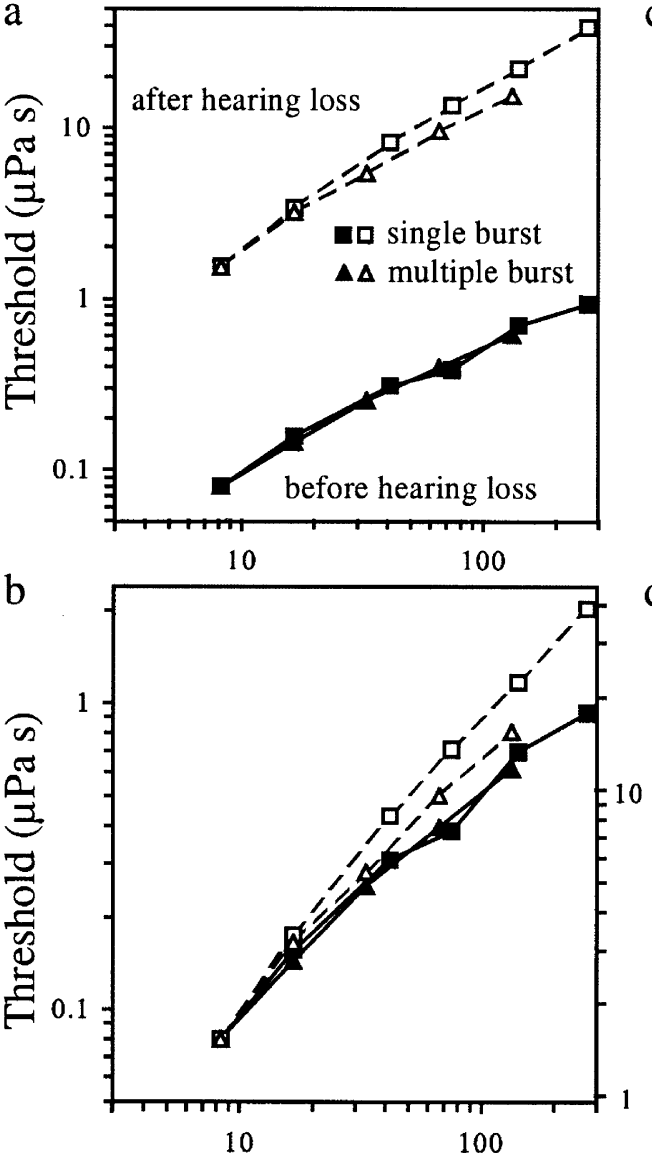

Stimulus duration (ms)

$T\left(t_{\text {eff }}\right)=\int_{0}^{t_{\text {eff }}} P_{\text {eff }}^{1}(t) d t$

0

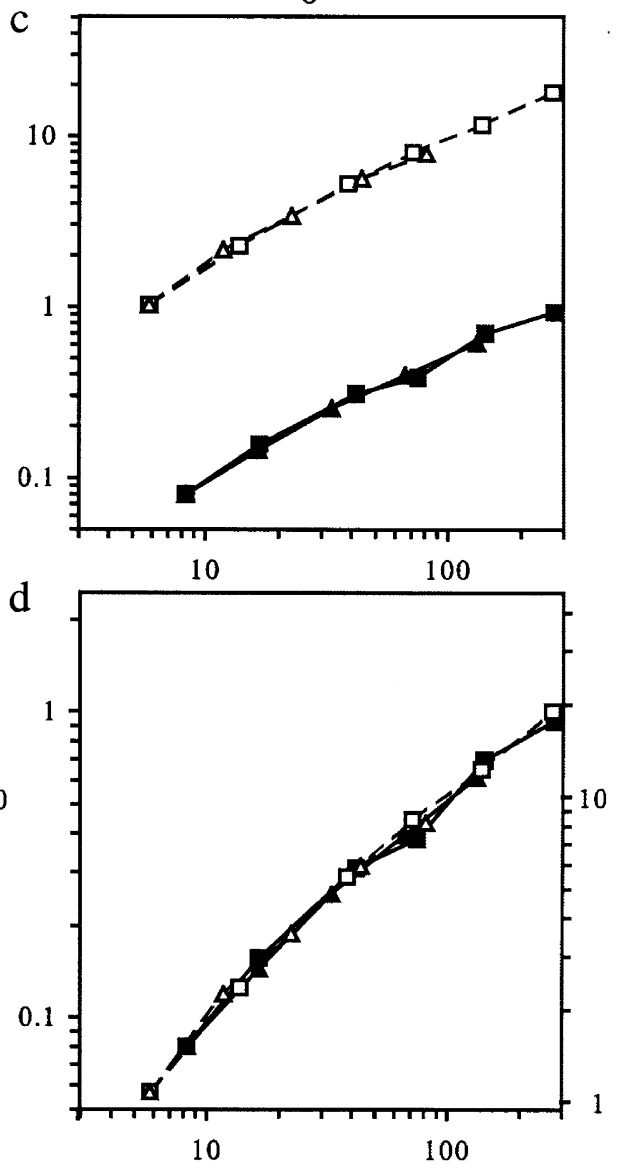

Eff. stim. duration (ms)
FIG. 2. Grand mean thresholds specified in terms of integrated $P(t)$ and plotted against physical stimulus duration $(\mathbf{a}, \mathbf{b})$, and specified in terms of integrated $P_{\text {eff }}(t)$ and plotted against effective stimulus duration $(\mathbf{c}, \mathbf{d})$. $P_{\text {ineff }}$ was $5 \mathrm{nPa}$ before and $77 \mu \mathrm{Pa}$ after hearing loss. In $\mathbf{b}, \mathbf{d}$ thresholds before (left ordinates) and after (right ordinates) hearing loss are shifted relative to each other, such that thresholds for the stimulus of the shortest duration (shortest effective duration before hearing loss in d) become superimposed. jects $P_{\text {ineff }}=0$, but $P_{\text {ineff }}$ might be so small that Eq. (1) suffices to describe the data. At least this is the case for the cats included in this study, as is shown below. After hearing loss, however, when $P_{\text {ineff }}$ clearly exceeds zero, it becomes more obvious that $P_{\text {ineff }}$ is a covert variable and that the two overt variables of Eq. (1), $k$ and $m$, attempt to compensate for it.

Figure 3 depicts this idea and illustrates its consequences for a multiple- $(n=4)$ and a single-burst stimulus. It uses values close to those obtained from the grand mean data (see below). Figure 3a illustrates the normal situation where $P_{\text {ineff }}$ is assumed to be zero and hence $P(t)=P_{\text {eff }}(t)$. The measured maximum amplitude of $P(t)$ for the multiple-burst threshold stimulus was $15.3 \mu \mathrm{Pa}$. The maximum amplitude of $P(t)$ for a single-burst threshold stimulus of the same physical duration and with the same onset and offset times should then be $8.7 \mu \mathrm{Pa}$ in order for the thresholds to be identical, when specified in terms of integrated $P(t)$ or $P_{\text {eff }}(t)$ (viz., $0.25 \mu \mathrm{Pa} \mathrm{s}$; gray areas in Fig. 3a). This corresponds to $56 \%$ of the maximum amplitude of the multiple-burst stimulus (dotted horizontal line). [The maximum amplitude of $P(t)$ actually obtained for the single-burst stimulus via interpolation from measured thresholds to stimuli of 16.64 and $41.6 \mathrm{~ms}$ was $9.5 \mu \mathrm{Pa}$, a deviation of $9 \%$ from the expected value (error bar in Fig. 3a)].

Figure $3 \mathrm{~b}$ illustrates the situation after hearing loss. The measured maximum amplitude of $P(t)$ for the multiple-burst threshold stimulus was $325 \mu \mathrm{Pa}$. Now, the model assumes some limit, $P_{\text {ineff }}$ (of 77 $\mu \mathrm{Pa}$ ), below which the sound pressure is unable to drive the system (white areas below dashed and continuous line in Fig. 3b). Consequently, the maximum amplitude of $P(t)$ for the single-burst stimulus must be $242 \mu \mathrm{Pa}$, or $75 \%$ of that for the multiple-burst stimulus, in order for thresholds to be identical, when specified in terms of integrated $P_{\text {eff }}(t) \quad(3.39 \mu \mathrm{Pa} \mathrm{s}$; gray areas in Fig. $3 \mathrm{~b})$. [The maximum amplitude of $P(t)$ actually obtained for the single-burst stimulus via interpolation from measured thresholds was $251 \mu \mathrm{Pa}$, a deviation of $4 \%$ from the expected value for 
before hearing loss

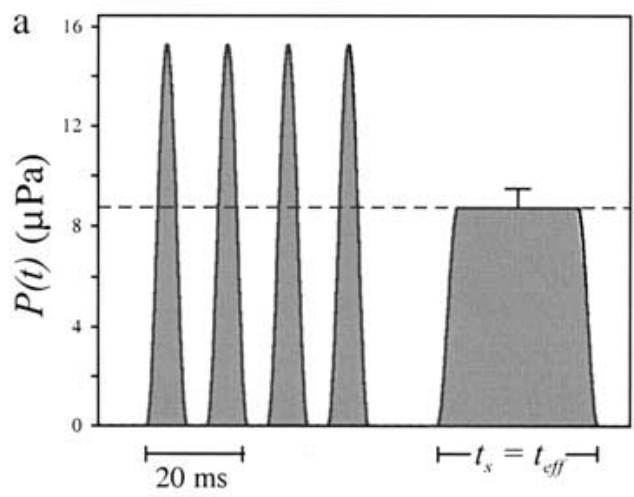

FIG. 3. Consequences of an elevated baseline and a reduced sensitivity for two threshold stimuli, a multiple- (stimulus 3, left) and a single-burst stimulus (right). Thresholds and parameters illustrated are close to those obtained from the grand mean data. a. Before hearing loss, $P_{\text {ineff }}$ is assumed to be zero. The multiple- and the single-burst stimuli illustrated are identical with respect to the area underneath $P(t)$ (gray area). The maximum amplitude of the singleburst threshold stimulus, which has the same physical duration ( $33.28 \mathrm{~ms}$ ) as the 4 bursts of the multiple-burst stimulus combined and the same onset and offset times $(4.16 \mathrm{~ms})$, equals 58 of that of the multiple-burst stimulus (dotted horizontal line). The maximum amplitude actually obtained for the single-burst stimulus, via interpolation from measured thresholds to stimuli of $16.64-$ and $41.6-\mathrm{ms}$ physical duration, was slightly larger, viz., 62.2 of that of the multiple-burst stimulus (error bar). b. After hearing loss, $P_{\text {ineff }}=77 \mu \mathrm{Pa}$. As long as $P(t)<P_{\text {ineff, }}$ it is unable to drive the system (white areas

$P_{\text {ineff }}=77 \mu \mathrm{Pa}$ (error bar in Fig. 3b), but a deviation of $27.1 \%$ from that expected for $P_{\text {ineff }}=0$.] Consequently, when thresholds are specified in terms of the temporal integral of $P(t)$, i.e., of the gray and white areas underneath the envelope combined, then the threshold for the single-burst stimulus is considerably higher than that for the multiple-burst stimulus of the same duration, qualitatively consistent with the data (Fig. 2a,b).

A second important consequence of the EBTS model concerns the stimulus duration. The stimulus can affect the system only when $P(t)>P_{\text {ineff. Hence, }}$ the effective stimulus duration $t_{\mathrm{eff}}$ is shorter than the physical duration $t_{\mathrm{s}}$ when $P_{\text {ineff }}>0$, unless the temporal envelope is rectangular. The difference between $t_{\mathrm{s}}$ and $t_{\text {eff }}$ depends on the magnitude of $P_{\text {ineff }}$ and on the shape of the temporal envelope. The single-burst stimulus in Figure $3 \mathrm{a}$ has a plateau duration of $24.96 \mathrm{~ms}$ and onset and offset times of $4.16 \mathrm{~ms}$ each. Hence, its total physical duration (of $33.28 \mathrm{~ms}$ ) matches that of the multiple-burst stimulus in that panel $(8 \times 4.16 \mathrm{~ms}=33.28 \mathrm{~ms})$. The two stimuli also match with respect to their effective duration (also $33.28 \mathrm{~ms}$ ), since $P_{\text {ineff }}=0$. However, if now $P_{\text {ineff }}$ increases, the effective duration of such a single-burst stimulus (which has 2 onset and offset after hearing loss

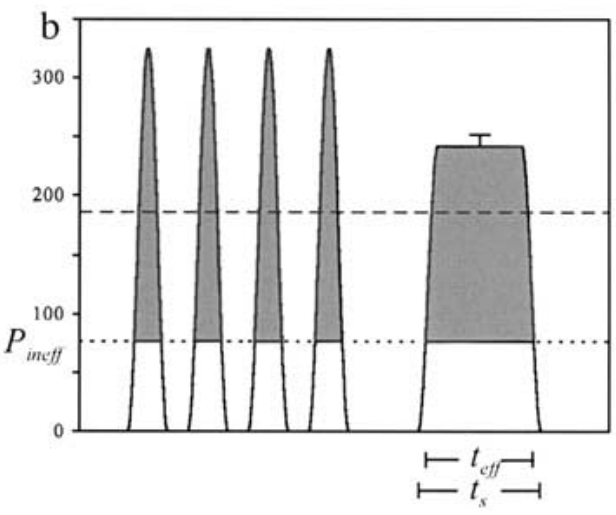

below dotted and continuous line). Consequently, the maximum amplitude must be 74.4 of that of the multiple-burst stimulus in order for thresholds to be identical when specified in terms of integrated $P_{\text {eff }}(t)$ [gray areas underneath $P(t)$; they are approximately 15 times larger than those in $\mathbf{a}$, reflecting the reduction in sensitivity]. The maximum amplitude obtained for the single-burst stimulus via interpolation from measured thresholds was slightly larger, viz., 77.4 of that of the multiple-burst stimulus (error bar). Also note that the effective stimulus duration $t_{\text {eff, }}$ given by the total time that $P(t)$ exceeds $P_{\text {ineff, }}$ is shorter than the physical stimulus duration $t_{\mathrm{s}}$ when $P_{\text {ineff }}>0$. The single-burst stimulus in $\mathbf{b}$ has a shorter physical duration, viz., $25.67 \mathrm{~ms}$, than the multiple-burst stimulus, viz. $33.28 \mathrm{~ms}$, but it has the same effective duration as the multiple-burst stimulus in b, viz., $22.51 \mathrm{~ms}$. The effective durations of both stimuli in $\mathbf{b}$ are shorter than those in $\mathbf{a}$, which are $33.28 \mathrm{~ms}$.

times) would decrease less than that of the multipleburst stimulus (which has 8 such times). Consequently, given the multiple-burst stimulus and a given increase in $P_{\text {ineff, }}$ a single-burst stimulus that matches the effective duration of the multiple-burst stimulus must have a plateau duration shorter than $24.96 \mathrm{~ms}$. Phrased differently, when $P_{\text {ineff }}>0$ and the multipleburst stimulus matches a single-burst stimulus in effective duration, the physical duration of the latter stimulus is shorter. This situation is illustrated in Figure 3b. The shorter physical duration of the single-burst stimulus in that panel is best seen by comparing it with that in Figure 3a.

\section{Quantitative tests of the EBTS model}

To test the EBTS model quantitatively, we calculated a measure of difference between the thresholds of the multiple- and the single-burst series, viz., the mean $(C V)^{2}$ (see Methods). Thresholds were specified in terms of the temporal integral of $P_{\text {eff }}(t)$, i.e., of the size of the shaded areas in Figure 3 when $P(t)>P_{\text {ineff. }}$ We then minimized the measure of difference by varying $P_{\text {ineff }}$ as the only free parameter and with the only constraint that $P_{\text {ineff }} \geq 0$. If $P_{\text {ineff }}=0$, then $P_{\text {eff }}(t)=P(t)$. Note that with this approach, $P_{\text {ineff }}$ is 
fitted such that thresholds, specified in terms of the temporal integral of $P_{\text {eff }}(t)$, to stimuli of the singleand multiple-burst series match as closely as possible across all effective durations. It is not fitted to yield values of the power $m$ that would be as similar as possible after compared to before hearing loss.

An elevated baseline accounts for the mismatch of thresholds to single- and multiple-burst stimuli

Figure 2c illustrates for the grand mean data that thresholds, when specified in terms of the temporal integral of $P_{\text {eff }}(t)$, to the single- and multiple-burst stimuli remain very similar before hearing loss (filled symbols) and indeed become very similar in the same animals after hearing loss (open symbols). The symbols in Figure 2c are located more leftward than those in Figure 2a, because the effective stimulus duration $t_{\text {eff }}$ is shorter than the physical duration $t_{\mathrm{s}}$ when $P_{\text {ineff }}>0$ (cf. Fig. 3b). This is most obvious for the open symbols and for short durations.

Figure $4 \mathrm{a}, \mathrm{b}$ shows the improvement in the match of thresholds for single- and multiple-burst stimuli which is achieved by adding an optimal $P_{\text {ineff. The }}$ panels show the measure of difference, mean $(C V)^{2}$, for each cat individually and for the grand mean data, before (Fig. 4a) and after (Fig. 4b) hearing loss. Before hearing loss, the improvement in match is negligible (the lines in Fig. 4a are essentially horizontal). Furthermore, the measure of difference for the grand mean data is considerably smaller than for any of the individuals. This suggests that the mismatch of thresholds in individuals is largely due to unsystematic deviations, which average out but which cannot be compensated for by $P_{\text {ineff. In contrast, after hear- }}$ ing loss, the improvement in the match of thresholds achieved by adding this parameter is substantial, in both individuals and the grand mean data (Fig. 4b). In the latter, the measure of difference decreases by more than an order of magnitude. Furthermore, without $P_{\text {ineff }}$ that measure for the grand mean data falls within the range of measures for the individuals. This indicates that the mismatch of thresholds in the individuals, and in the grand mean data, is due to systematic differences, which do not average out. However, these systematic differences can be compensated for by $P_{\text {ineff: }}$ With this parameter the mismatch of thresholds in the grand mean data after hearing loss is smaller than in any individual cat and is as small as in the grand mean data before hearing loss (cf. Fig. 4a,b). The measures of difference in the individual cats before hearing loss, where they largely reflect unsystematic deviations, with or without $P_{\text {ineff }}$, are about as large as after hearing loss without $P_{\text {ineff }}$, where they reflect the sum of unsystematic deviations and of the systematic differences between thresholds
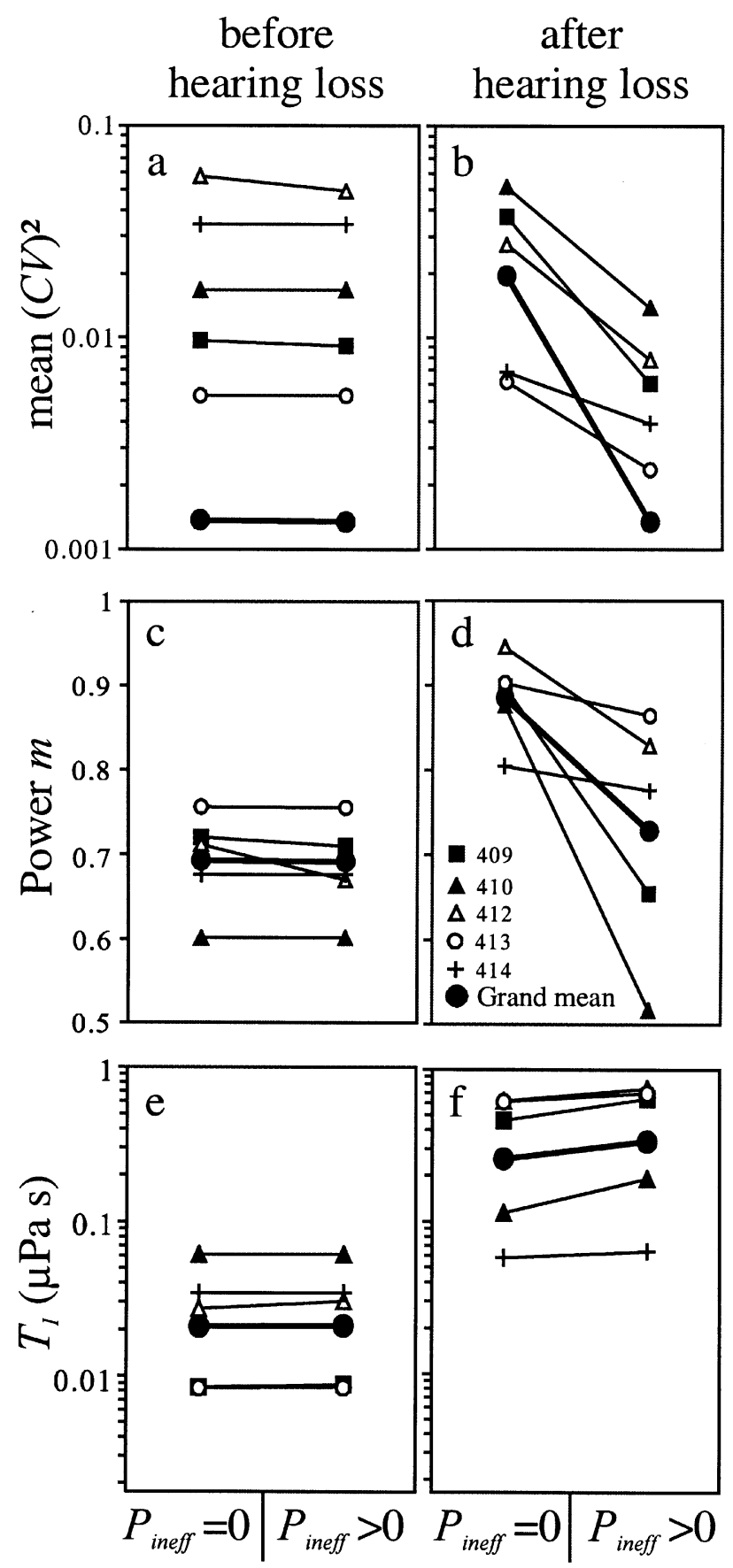

FIG. 4. Consequences of fits of an altered baseline $\left(P_{\text {ineff }}\right)$. Data points from a given individual or from the grand mean (key in $\mathbf{d}$ applies to all panels) are connected. In each panel the left data points are for $P_{\text {ineff }}=0$, i.e., with thresholds specified in terms of integrated $P(t)$, and the right data points are for $P_{\text {ineff }} \geq 0$, i.e., when thresholds are specified in terms of integrated $P_{\text {eff }}(t)$. The left and right columns show the effects of $P_{\text {ineff }}$ before and after hearing loss, respectively. $\mathbf{a}, \mathbf{b}$. Measure of difference, mean $\left(\mathrm{CV}^{2}\right.$, of thresholds to stimuli of the single- and the multiple-burst series. $\mathbf{c}, \mathbf{d}$. Power $m$ of the increase in thresholds, specified in terms of integrated $P(t)$ or $P_{\text {eff }}(t)$, with physical or effective stimulus duration, respectively. The values were obtained from fits of Eqs. (1) and (2), respectively, with $P_{\text {ineff }}$ set to that value which produced the closest match of thresholds to the stimuli from the single- and multiple-burst series in a given data set. e,f. Extrapolated thresholds for a 1-ms duration signal, $T_{1}$, obtained from the same fits. Conventions as in $\mathbf{c}, \mathbf{d}$. For explanations see text. 
for stimuli of the single- and multiple-burst series. This suggests that the unsystematic deviations before hearing loss are larger than thereafter. This may be explained, for example, by the presence of some ambient low-level noise or sounds generated in the context of the experimental procedures, which would affect threshold estimates more when the cats' sensitivities are high than when they are low.

\section{An elevated baseline accounts for the change in slope of the temporal integration function}

Figure $2 d$ replots the threshold data of Figure 2c, but as in Figure $2 \mathrm{~b}$, the data points are shifted relative to each other, now by a factor of 17.9 , such that the thresholds of normal-hearing and hearing-impaired cats are superimposed for $8.24 \mathrm{~ms}$. This is the effective duration of the shortest stimulus before hearing loss. Again, the overall reduction in sensitivity due to the trauma can still be seen by comparing the numbers on the two ordinates. The main purpose of the shift is to demonstrate that the increase in thresholds, when specified in terms of the temporal integral of $P_{\text {eff }}(t)$ rather than of $P(t)$, with effective stimulus duration $t_{\mathrm{eff}}$ is remarkably similar before (left ordinate) and after hearing loss (right ordinate). The nearly perfect superposition of the thresholds over the entire range of effective durations is striking, even though the thresholds before and after hearing loss were purposely superimposed at only a single, rather short duration.

This close match then suggests that when these data are fitted with Eq. (2), rather than with Eq. (1), estimates of the power $m$ obtained before and after hearing loss should be rather similar. Figure 4c,d shows that this is indeed the case. Before hearing loss, the power $m$ is largely unaffected by this extension of the model (note that the lines in Fig. 4c are essentially horizontal), although $m$ decreases slightly for 3 of the 5 cats, viz., those for which $P_{\text {ineff }}>0$ (see below), but appreciably only in cat 412 . After hearing loss, the decrease in $m$ is substantial in every individual as well as in the grand mean data. When fitted with Eq. (1), the estimates of $m$ are all larger after than before hearing loss. In contrast, when fitted with Eq. (2), they overlap, although they scatter more widely after than before hearing loss. The number of degrees of freedom of the model is identical before and after hearing loss. However, before hearing loss $P_{\text {ineff }}$ has very little influence on the estimation of the other two parameters (viz., $k$ and especially $m$ ), because $P_{\text {ineff }}$ is so small (see below and Fig. 5). After hearing loss, the influence becomes considerably larger and, therefore, the uncertainty in determining $m$ also increases (see below and also Fig. 6). This likely accounts for the wider scatter of the estimated values of $m$ after hearing loss and with $P_{\text {ineff. }}$
For the grand mean data, the value of $m$ obtained by fits of Eq. (2) after hearing loss, viz., $0.73 \pm 0.03$, is slightly larger than that obtained before hearing loss, viz., $0.69 \pm 0.03$. This insignificant difference in the estimates of $m$, despite the excellent superposition of the thresholds (Fig. 2d), probably results from the slight downward curvature of the function relating threshold to duration in double-log coordinates in this particular set of data (see above and Fig. 2). This is so because a unidirectional movement of all points toward the steeper sloping end will lead to a steeper slope of a straight-line fit. As explained above, the movement is a consequence of the replacement of $P(t)$ by $P_{\text {eff }}(t)$ and of $t_{\mathrm{s}}$ by $t_{\text {eff. }}$.

\section{The change in gain}

The extension of our previous model [Eq. (1)] to the EBTS model [Eq. (2)] has only small effects on the estimates of the sensitivity, as captured by parameter $k$ in Eq. (1) and (2). This is illustrated in Figure 4e,f, which plots extrapolated thresholds $T_{1}$ (in $\mu \mathrm{Pa}$ s), i.e., $T\left(t_{\mathrm{s}}\right)$ or $T\left(t_{\mathrm{eff}}\right)$ for $t_{\mathrm{s}}$ or $t_{\text {eff }}$ of $1 \mathrm{~ms}$. Before hearing loss, all lines are essentially horizontal (Fig. 4e). After hearing loss, cats are less sensitive than before and these differences, unlike those in power $m$, are not compensated for by $P_{\text {ineff. }}$ On the contrary, the lines in Figure $4 \mathrm{f}$ have small positive slopes, i.e., the differences in $T_{1}$ before and after hearing loss are slightly larger with than without that parameter.

\section{Magnitudes of changes in baseline and gain}

Figure 5 a plots the estimates of $P_{\text {ineff, }}$, derived from the fits of Eq. (2) to data from individual cats and the grand mean, before and after hearing loss. $P_{\text {ineff }}$ increases for every cat as well as for the grand mean data. For the latter, $P_{\text {ineff }}$ increases by more than 4 orders of magnitude! Before hearing loss, the fits yield estimates for $P_{\text {ineff }}$ of $0 \mathrm{~Pa}$ for 2 of the 5 cats (viz., cats 410 and 414), and values rather close to zero for the other 3 cats (viz., $1.5 \mathrm{nPa}$ for cat 413, 46 $\mathrm{nPa}$ for cat $409,1 \mu \mathrm{Pa}$ for cat 412), and $5 \mathrm{nPa}$ for the grand mean data. After hearing loss, estimates of $P_{\text {ineff }}$ are $1.9 \mu \mathrm{Pa}(-23 \mathrm{~dB} \mathrm{SPL})$ for cat 414 and fall in the range of $44-210 \mu \mathrm{Pa}(3-17 \mathrm{~dB}$ SPL) for the other cats, and are $77 \mu \mathrm{Pa}$ (9 dB SPL) for the grand mean data.

Figure $5 \mathrm{~b}$ plots the corresponding estimates of $T_{1}$ before and after hearing loss. $T_{1}$ also increases, i.e., sensitivity decreases, for every cat as well as for the grand mean data. For the grand mean data, $T_{1}$ increases by 1-2 orders of magnitude, about 3 orders of magnitude less than $P_{\text {ineff. }}$ Consequently, the ratio of $P_{\text {ineff }}$ to $T_{1}$, a critical parameter (see Figs. 6 and 10 and associated text), increases by about 3 orders of 

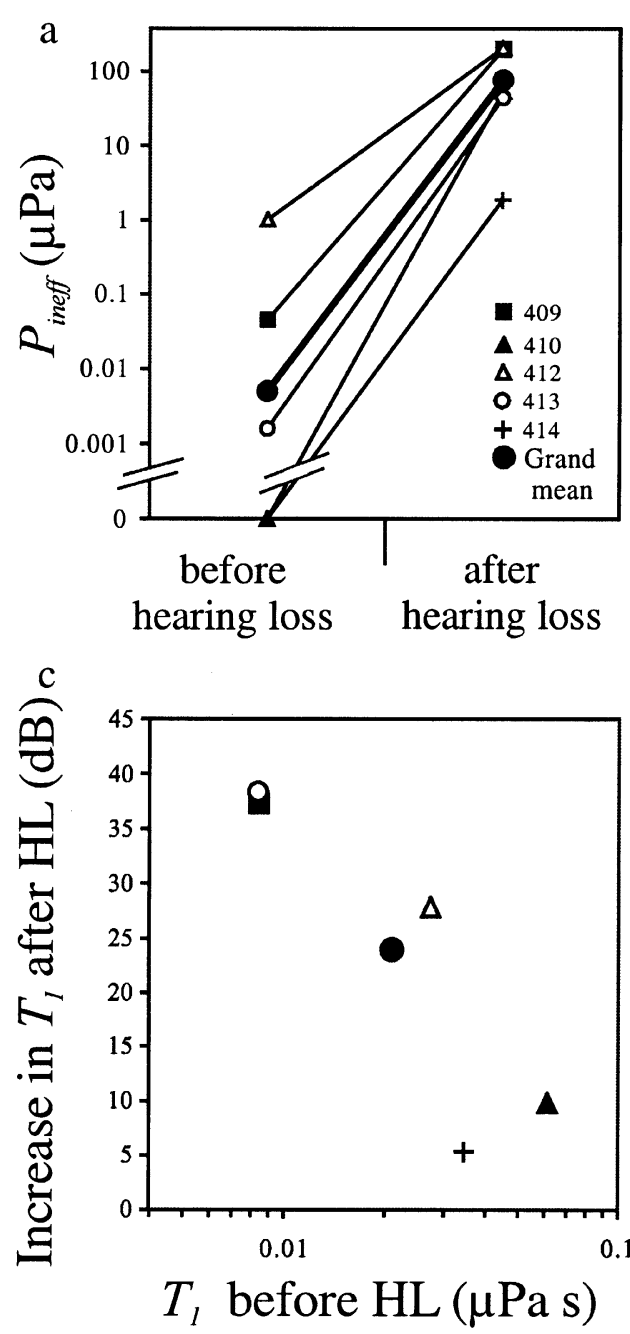

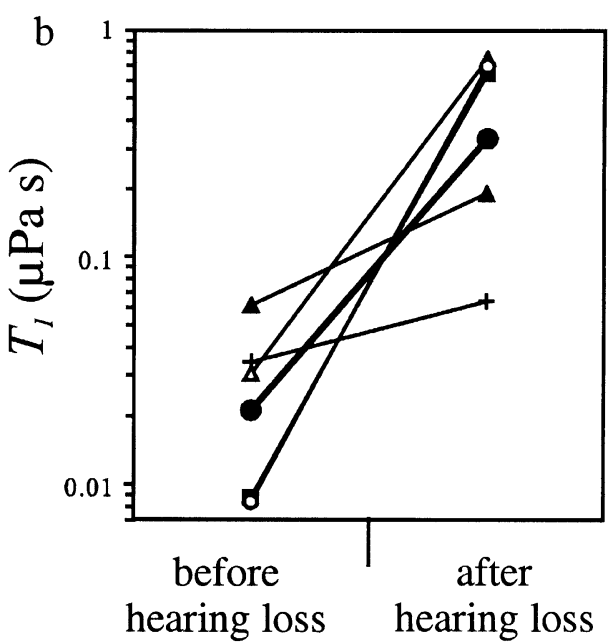

d

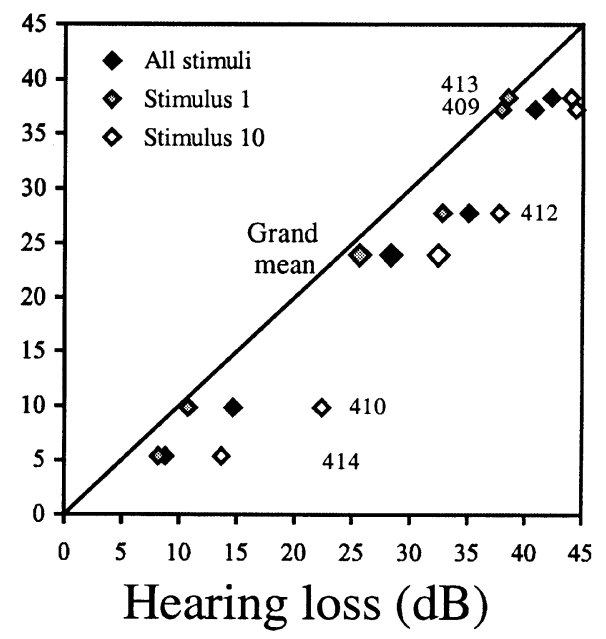

FIG. 5. Changes in baseline (a) and sensitivity (b) as a consequence of noise trauma. Data points from a given individual or the grand mean are connected (key in $\mathbf{a}$ applies also to $\mathbf{b}$ and $\mathbf{c}$ ). In each panel the left and right data points show the estimates of $P_{\text {ineff }}(\mathbf{a})$ and of $T_{1}(\mathbf{b})$ before and after hearing loss, respectively. c. The increase in $T_{1}$ (in $\mathrm{dB}$ ) due to the noise trauma (48-h exposure to a $110 \mathrm{~dB}$ SPL 2-kHz tone) is the larger the lower an individual's $T_{1}$, i.e., the higher its sensitivity, before the trauma. $\mathbf{d}$. The increase in $T_{1}$ (in $\mathrm{dB}$ ) plotted against the hearing loss (in $\mathrm{dB}$ ) for the shortest stimulus (1), the longest stimulus (10), and the average across all 10 tested stimuli. Note that the increase in $T_{1}$ correlates strongly with hearing loss but does not fully account for it, the less so the longer the duration of the test stimulus. magnitude. Interestingly, and as shown in Figure 5c, the increase in $T_{1}$ (in $\mathrm{dB}$, i.e., as 20 times the logarithm of the ratio of $T_{1}$ before to after hearing loss) induced by the trauma tended to be the smaller the lower the logarithm of $T_{1}$ before hearing loss (Pearson rank correlation coefficient $R=-0.877 ; n=5$ ). This may not be coincidental, because the trauma was induced by exposure to a $110 \mathrm{~dB}$ SPL $2-\mathrm{kHz}$ tone, so that the sensation level (SL) of this stimulus, and presumably also its traumatic consequences, would be the smaller the lower an individual's sensitivity. Figure $5 \mathrm{~d}$ plots the increase in $T_{1}$ (in $\mathrm{dB}$ ) against the hearing loss for each cat individually as well as for the grand mean data. The hearing losses, i.e., the differences in threshold SPLs after and before the trauma, are shown for the shortest stimulus (1, gray diamonds), the longest stimulus (10, open diamonds) and for the average across all 10 tested stimuli (black diamonds). Figure $5 \mathrm{~d}$ illustrates that for all conditions the increase in $T_{1}$ is strongly correlated with the hearing losses $(R=0.992,0.990$, and 0.995 for stimulus 1,10 , and the average, respectively; $n=5$ in each case). It also illustrates that the increase in $T_{1}$ captures most of the hearing losses, as conventionally defined. This holds especially for the shortest stimulus (note that the data points fall rather close to the diagonal). However, for the longest stimulus, data points fall some $10-15 \mathrm{~dB}$ to the right of the diagonal, and the data points for the average hearing loss across all stimuli fall in between those for the shortest and the longest stimulus. This reflects the fact that as stimulus duration increases, the contribution of $P_{\text {ineff }}$ to the total hearing loss also increases. However, that contribution varies little between cats (3.5-7.2 dB for the average across all stimuli) compared to the variation in hearing losses $(8.8-40.8 \mathrm{~dB}$, also for the average across stimuli; Fig. 5d). Unsurprisingly therefore, the increases in $P_{\text {ineff }}$ and $T_{1}$ were uncorrelated (not shown).

The above analyses are thus consistent with our view that the noise trauma has had two different consequences, one which affected the sensitivity $k$, i.e., a simple gain, and one which affected $P_{\text {ineff }}$, i.e., the baseline above which sound pressure is effective. 


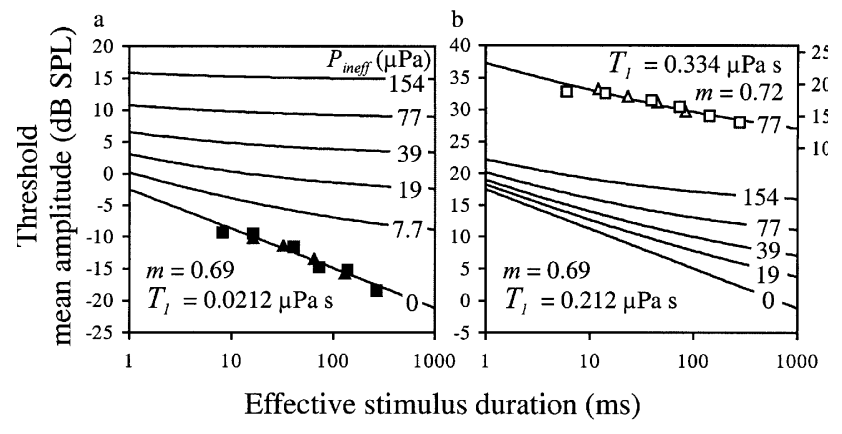

FIG. 6. Predicted effects of $P_{\text {ineff }}$ and $T_{1}$ on hearing loss and on shape of temporal integration functions for (theoretical) stimuli with rectangular temporal envelopes. Here physical and effective duration are identical. In $\mathbf{a}, T_{1}$ is a factor of 10 smaller (i.e., sensitivity is higher) than it is in $\mathbf{b}$. The parameter is $P_{\text {ineff }}$ (numbers on curves are in $\mu \mathrm{Pa}$ ). Note that with $T_{1}$ fixed ( $m$ is also fixed at 0.69), the hearing loss increases as $P_{\text {ineff }}$ increases. The loss depends on stimulus duration, causing a change in the shape and a decrease in average slope of the temporal integration functions. The magnitude of the loss with respect to $P_{\text {ineff }}=0$ and the change in shape produced by a given $P_{\text {ineff }}$ are determined by the ratio of $P_{\text {ineff }}$ to $T_{1}$. Note that the functions for $P_{\text {ineff }}=7.7 \mu \mathrm{Pa}$ in $\mathbf{a}\left(P_{\text {ineff }} /\left(T_{1}=363^{\mathrm{s}-1}\right)\right.$ and for $P_{\text {ineff }}=77$ $\mu \mathrm{Pa}$ in $\mathbf{b}\left(P_{\text {ineff }} /\left(T_{1}=363^{\mathrm{s}-1}\right)\right.$ have identical shapes and are displaced upward by the same amount from the functions for $P_{\text {ineff }}=0$. The solid and open symbols on the bottom function in $\mathbf{a}$ and on the top function in $\mathbf{b}$ represent the grand mean thresholds of the cats before and after hearing loss, respectively. Data points are replotted from Figure $1 \mathrm{~b}$ (same key as there), with the difference that here the SPLs correspond to the mean amplitude of each stimulus and duration is the effective stimulus duration. The top function in $\mathbf{b}$ (right ordinate applies) shows the function ideally expected for stimuli of rectangular envelopes and with the parameters obtained from the grand mean data of the cats after hearing loss $\left(T_{1}=0.334 \mu \mathrm{Pa} \mathrm{s} ; P_{\text {ineff }}=77\right.$ $\mu \mathrm{Pa} ; m=0.72)$.

Despite a common cause, viz., the noise exposure, the two consequences may be reasonably independent, although of course we cannot exclude the possibility that with a larger sample some correlation may have emerged. Such a correlation is suggested by studies of, for example, Elliott (1963), Wright (1968), Brahe Pedersen and Elberling (1973), and Brahe Pedersen and Salomon (1977) who found, in large samples of human subjects with sensorineural hearing losses, that the amount of temporal integration was the smaller the larger the hearing loss.

\section{Interactions of changes in gain and baseline in capturing hearing loss}

The sensitivity of the fits to those two model parameters which appear to be changed by the trauma, i.e., $k$ or $T_{1}$ and $P_{\text {ineff, }}$ and the interactions between them can be judged by inspection of Figure 6. The two panels show temporal integration functions as they would ideally be recorded with stimuli of rectangular envelopes. For $P_{\text {ineff }}=0$, these are straight lines in double-log plots of amplitude and duration with a slope (in $\mathrm{dB}$ per decade of duration) of $20(m-1)$. This follows readily by dividing both sides of Eq. (1) by $t_{\mathrm{s}}$ (see also Heil and Neubauer 2003). The straight line in Figure 6a is based on the values of $T_{1}$ and of $m$ which were obtained by fitting Eq. (l) to the grand mean data. Conventional temporal integration functions tend to be curved, being the steeper the shorter the stimulus duration. This curvature is likely to result from the concomitant increase in the proportion of onset and offset times with respect to the entire stimulus duration, in combination with the fact that the SPLs plotted correspond to the maximum amplitude of each stimulus. When instead SPLs are plotted that correspond to the mean amplitude of each stimulus, then the data points tend to fall on a straight line, which is illustrated in Figure 6a. Here, the grand mean thresholds of the cats before hearing loss are replotted from Figure 1b (data points on straight line in Fig. 6a; symbols as in Fig. 1b), with the difference being that in Figure 1b the SPLs correspond to the maximum, and in Figure 6a they correspond to the mean amplitude of each stimulus. This operation is equivalent to replacing the actual stimulus with one of a rectangular envelope with identical physical duration and temporal integral of the pressure envelope as the actual stimulus.

Each panel of Figure 6 illustrates the effect of $P_{\text {ineff }}$ on temporal integration functions. In each panel, temporal integration functions are shown for different values of $P_{\text {ineff }}$ (see numbers on lines). As $P_{\text {ineff }}$ increases, with other parameters held constant, threshold SPL increases, but the more so the longer the stimulus. Consequently, temporal integration functions become less steep. They also change shape, being curved rather than straight when $P_{\text {ineff }}>0$. The magnitude of the threshold increase with respect to $P_{\text {ineff }}=0$ and the change in shape produced by a given $P_{\text {ineff }}$ depend on the value of $T_{1}$. This is evident by comparing Figure $6 \mathrm{a}$ with $\mathrm{b}$, since each panel plots temporal integration functions for a different value of $T_{1}$. In fact, both effects are solely determined by the ratio of $P_{\text {ineff }}$ and $T_{1}$. Note that the functions for $P_{\text {ineff }}=7.7 \mu \mathrm{Pa}$ in Figure $6 \mathrm{a}\left(T_{1}=0.0212 \mu \mathrm{Pa} \mathrm{s}\right)$ and for $P_{\text {ineff }}=77 \mu \mathrm{Pa}$ in Figure $6 \mathrm{~b} \quad\left(T_{1}=0.212 \mu \mathrm{Pa} \mathrm{s}\right)$ have identical shape and are displaced upward by the same amount from the functions for $P_{\text {ineff }}=0$. These analyses show that the final shape of a temporal integration function is determined by the specific combination of $T_{1}$ and $P_{\text {ineff. Only when } P_{\text {ineff }}=0 \text { is }}$ the function shape independent of $T_{1}$. The upper line in Figure $6 \mathrm{~b}$ shows the function ideally expected for stimuli of rectangular envelopes and with the parameters obtained from the grand mean data of the cats after hearing loss. The open symbols represent the grand mean thresholds of the cats after hearing loss, replotted from Figure 1b, but specified 
in terms of the SPL that corresponds to the mean amplitude of each stimulus (key as in Fig. 1b).

It is thus clear that a variety of shapes of temporal integration functions can be produced by an adequate selection of $T_{1}$ and $P_{\text {ineff. However, as stated }}$ above, for comparison with Figure 6, threshold SPLs from the literature would first have to be converted into SPLs that correspond to the mean amplitude of each stimulus.

An alternative model: noise trauma might change the quantity being integrated

It is conceivable that the noise trauma may have caused a change in the exponent $q$ of $P(t)$ within the integrand of Eq. (1). It has been suggested that, prior to integration, the quantity being integrated might be subject to some power-law nonlinearity (Penner 1978; Oxenham et al. 1997; Moore 1991, 1996; Moore and Oxenham 1998). For example, if the true quantity being integrated were $I(t)$, which is proportional to $P^{2}(t)$, but prior to integration were subject to a compressive nonlinearity of power 0.5 , it might appear as if $P(t)$ were integrated, since $\left[P^{2}(t)\right]^{0.5}=P(t)$. Also, if hearing loss would result in altered compression, then the optimal exponent $q$ would be affected. In the above example, a complete loss of compression would lead to an increase of the exponent $q$ from 1 to 2 . We have tested this idea quantitatively by calculating the measure of difference, mean $(C V)^{2}$, of the thresholds for the single- and multiple-burst stimuli, now specified as the temporal integral of $P^{q}(t)$, for different values of the exponent $q$ and then plotted the measure of difference as a function of $q$. $P_{\text {ineff }}$ was set to zero.

Figure 7 shows the results obtained for the grand mean thresholds. Before hearing loss, the measure of difference shows a clear minimum, i.e., the match is best at $q=0.97$, a value very close to 1 (Fig. 7a, filled circles). This result strongly confirms our previous conclusion (Heil and Neubauer 2003). After hearing loss, the measure of difference reaches a minimum at a value of $q$ that is indeed larger than before, as predicted by a loss of compression. However, the minimum occurs at $q=2.53$ (Fig. $7 \mathrm{a}$, open circles). Thus, if the true quantity being integrated were $I(t)$, one would have to assume an expansive nonlinearity after hearing loss. The value of mean $(C V)^{2}$ at this minimum is approximately 4 times larger than at $q=1$ before hearing loss (Fig. 7a). The factor is even larger (viz., approximately 6 ) at $q=2$, the value expected if the true quantity being integrated were $I(t)$ and hearing loss resulted in a complete loss of compression but not resulted in expansion. This is more clearly shown in Figure $7 \mathrm{~b}$, which plots various measures of difference for $q=2$ and $q=1$ normalized to
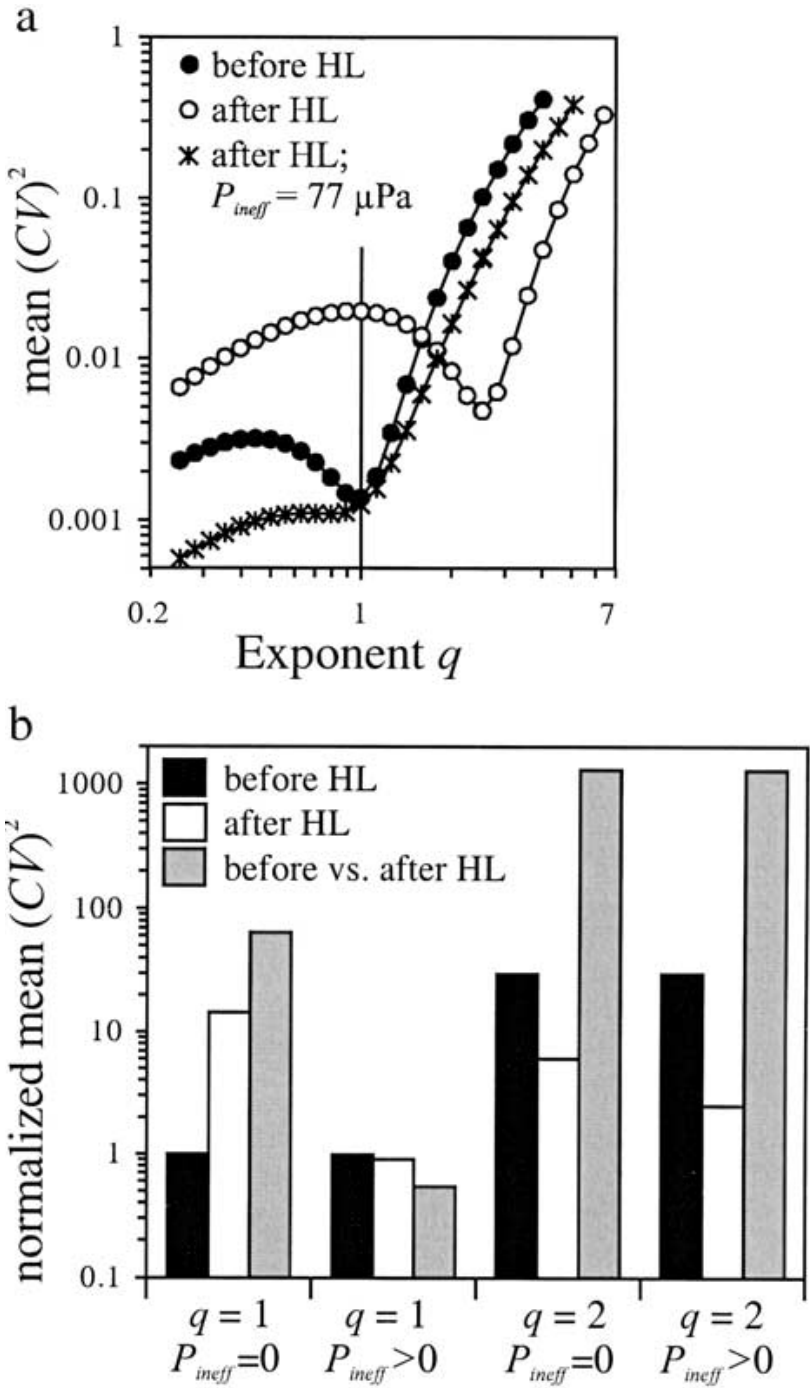

FIG. 7. Comparison of models. a. Measure of difference, mean $\left(\mathrm{CV}^{2}\right.$, of grand mean thresholds to stimuli of the single- and multipleburst series, expressed in terms of integrated $P^{q}(t)$ and plotted as a function of $q$. The measure is smallest, i.e., thresholds match best, for $q$ $\approx 1$ before (filled circles) and for $q \approx 2.5$ after (open circles) hearing loss. However, with $P_{\text {ineff }}=77 \mu \mathrm{Pa}$, the function after hearing loss (crosses) is similar to that before hearing loss for $P_{\text {ineff }}=0$. b. Measures of difference, mean $\left(\mathrm{CV}^{2}\right.$, normalized with respect to the condition of $q=1$ and $P_{\text {ineff }}=0$ before hearing loss (leftmost black bar). Black and open bars represent the normalized mean $\left(\mathrm{CV}^{2}\right.$ before and after hearing loss, respectively. Gray bars represent that measure between conditions, after the thresholds have been optimally superimposed (see text). Thresholds match as good as or even slightly better than they do for $q=1$ and $P_{\text {ineff }}=0$ before hearing loss when $q=1$ and $P_{\text {ineff }}$ is set to $5 \mathrm{nPa}$ before hearing loss (black bar second from left), or when $q=1$ and $P_{\text {ineff }}$ is set to $77 \mu \mathrm{Pa}$ after hearing loss (open bar second from left). Thresholds with $q=1$ and $P_{\text {ineff }}$ set to $5 \mathrm{nPa}$ before and $77 \mu \mathrm{Pa}$ after hearing loss can also be superimposed so that they match that well (gray bar second from left). When $q=1$ and $P_{\text {ineff }}$ is set to zero, thresholds after hearing loss do not match as well as before hearing loss, nor can thresholds before and after hearing loss be superimposed well (left block of bars). When $q=2$, i.e., when thresholds are specified in terms of integrated $I(t)$, and $P_{\text {ineff }}$ is either set to zero (second block of bars from right) or is set to its optimum $>0$ (right block of bars), thresholds before or after hearing loss do not even nearly match as well as they do before hearing loss when $q=1$ and $P_{\text {ineff }}=0$, nor can thresholds before and after hearing loss be superimposed well. 
that at $q=1$ and $P_{\text {ineff }}=0$ before hearing loss (leftmost column). Consequently, with the assumption of an altered exponent $q$, the match of thresholds to stimuli of the single- and multiple-burst series appears much worse after than before hearing.

How does a model of an altered exponent compare with that of an elevated baseline? Albeit after hearing loss the match of thresholds to stimuli of the single- and multiple-burst series appears reasonably good for $q=2.53$ (not shown) as well as for $q=2$ (Fig. 8a,b, open symbols), it is much worse (about 4 and about 7 times for $q=2.53$ and 2, respectively) than for $q=1$ and $P_{\text {ineff }}$ at its optimal value of $77 \mu \mathrm{Pa}$. These comparisons clearly favor the model of an elevated baseline. Furthermore, with the assumption of an altered $P_{\text {ineff }}$, the match of thresholds to stimuli of the single- and multiple-burst series is as good after hearing loss, with $P_{\text {ineff }}=77 \mu \mathrm{Pa}$, as it is before, whether $P_{\text {ineff }}$ is assumed to be zero (Fig. $7 \mathrm{a}, \mathrm{b}$ ) or is set to its optimal value of $5 \mathrm{nPa}$ (Fig. 7b). In contrast, with the assumption of an altered exponent $(q=2.53)$, alignment is about 4 times worse after than before hearing loss, as explained above. Also, with the model of an elevated baseline, thresholds before and after hearing loss can be specified in the same way and in the same physical units, viz., as the temporal integral of $P_{\text {eff }}(t)$ (in Pa s) (see Fig. 2), whereas an altered exponent $q$ would require thresholds to be specified in different physical units before and after hearing loss.

The assumption of an altered baseline in combination with $q=2$ also improves the alignment of thresholds somewhat, albeit only after hearing loss, since before hearing loss estimates of $P_{\text {ineff }}$ were zero. Nevertheless, the alignments achieved are much worse than with $q=1$, both before and after hearing loss (Fig. 7b).

Additional evidence for an altered baseline in combination with $q=1$, and against $q=2$, comes from the degree to which thresholds before and after hearing loss can be superimposed, i.e., from the match which can be achieved by parallel shifts of the threshold (on a logarithmic axis) versus duration functions [see Fig. 2b,d for $q=1$ without $P_{\text {ineff }}(\mathrm{b})$ and with $P_{\text {ineff }}(\mathrm{d})$, and Fig. 8b,d for $q=2$ without $P_{\text {ineff }}(\mathrm{b})$ and with $\left.P_{\text {ineff }}(\mathrm{d})\right]$. We quantified this match by first averaging the logarithms of the (interpolated) thresholds to the single- and multipleburst stimuli of the same duration, separately before and after hearing loss. We then superimposed these average thresholds for the stimuli of the shortest duration, as explained above, and then calculated the measure of difference, mean $(C V)^{2}$, between them. These measures were then also normalized with respect to the measure of difference of thresholds to the single- and multiple-burst stimuli before hearing loss for $q=1$ and $P_{\text {ineff }}=0$. Figure $7 \mathrm{~b}$ shows that these normalized measures of difference between conditions are 1300 for $q=2$ and 64 for $q=1$, when $P_{\text {ineff }}=0$. When the values of $P_{\text {ineff }}$ are set to their optimal estimates, the normalized measures of difference between conditions are 1281 for $q=2$ and 0.54 for $q=1$, consistent with the visual impression of a pronounced mismatch for $q=2$ (Fig. 8d) and an excellent match for $q=1$ (Fig. 2d). Thus, only with $q=1$ and $P_{\text {ineff }}>0$ is the match of thresholds between conditions as good as between thresholds to the single- and multiple-burst series within each condition.

\section{Consequences of an elevated baseline and temporal summation for suprathreshold stimuli}

Effective stimulus growth. Figure 9a shows four stimuli of rectangular temporal envelope, two each with durations of 300 and $8 \mathrm{~ms}$. The two stimuli labeled 0 dB SL have amplitudes that correspond very closely to those extrapolated from the grand mean thresholds of the cats after hearing loss. The value of $P_{\text {ineff }}=77$ $\mu \mathrm{Pa}$ also equals that obtained from these data. Figure $9 \mathrm{a}$ shows that with a given increment in stimulus level, here $2 \mathrm{~dB}$, the increment in the effective stimulus $T_{\text {eff }}$, specified as the temporal integral of $P_{\text {eff }}(t)$ (gray areas), is larger than $2 \mathrm{~dB}$. Note that the ratio of the gray areas is larger than that of the total areas underneath the envelopes of the stimuli because $P_{\text {ineff }}$ does not change with level. Figure $9 \mathrm{~b}, \mathrm{c}$ shows this more quantitatively. Figure $9 \mathrm{~b}$ plots, on linear axes, $T_{\text {eff }}$ (in $\mu \mathrm{Pa}$ s) against the maximum amplitude of $P(t), P$ (in $\mu \mathrm{Pa}$ ), for stimuli of rectangular temporal envelope. The diagonal is for $P_{\text {ineff }}=0$ and the other parallel lines are for values of $P_{\text {ineff }}$ of $19,39,77$, and $154 \mu \mathrm{Pa}$ (from left to right). Figure $9 \mathrm{~b}$ illustrates that $T_{\text {eff }}$ grows linearly with pressure, irrespective of the magnitude of $P_{\text {ineff. }}$ When $P_{\text {ineff }}=0, T_{\text {eff }}=0$ only when $P=0$. However, when $P_{\text {ineff }}>0, T_{\text {eff }}=0$ as long as $P \leq P_{\text {ineff. }}$ Only when $P>P_{\text {ineff, }}$, is there a linear growth of $T_{\text {eff }}$ with pressure. Figure $9 \mathrm{c}$ replots the same functions in double-log coordinates. Now, the slope $s$ of the functions relating the logarithm of $T_{\text {eff }}$ and the logarithm of $P$ obeys the formula:

$$
s=P /\left(P-P_{\text {ineff }}\right)
$$

A constant slope of 1 results only when $P_{\text {ineff }}=0$. When $P_{\text {ineff }}>0$, the slope is steeper than 1 , the more so the smaller the difference between $P$ and $P_{\text {ineff. }}$ With increasing pressure, the curves for $P_{\text {ineff }}>0$ approach that for $P_{\text {ineff }}=0$.

Figure 10 provides independent experimental evidence that for the cats in this study the growth functions of $\log \left(T_{\text {eff }}\right)$ with SPL near absolute threshold are indeed considerably steeper after than before hearing 

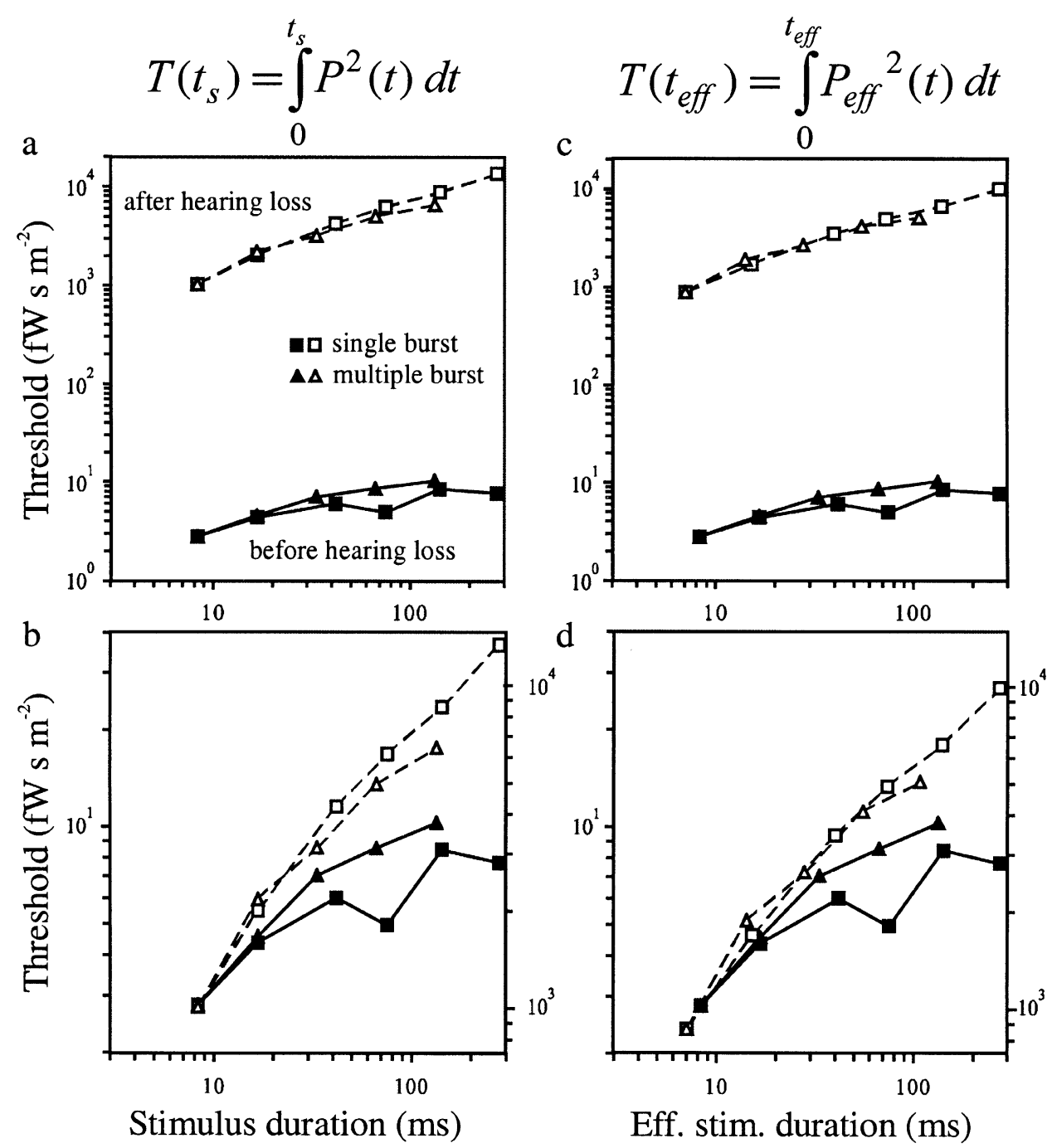

Eff. stim. duration (ms)

FIG. 8. Grand mean thresholds specified in terms of integrated $P^{2}(t)$, i.e., of energy density, and plotted against physical stimulus duration $(\mathbf{a}, \mathbf{b})$, and specified in terms of integrated $P_{\text {eff }}^{2}(t)$ and plotted against effective stimulus duration $(\mathbf{c}, \mathbf{d})$. Other conventions as in Figure 2.

loss. The evidence is based on an analysis of the SDs associated with the 12 accepted thresholds for each stimulus. Figure 10 plots the mean of the SDs across all 10 stimuli against the ratio of $P_{\text {ineff }}$ to $T_{1}$ (in s ${ }^{-1}$ ). As was explained above (Fig. 6), that ratio determines the shape of the temporal integration function, as well as the shape of the $\log \left(T_{\text {eff }}\right)$ vs. SPL function. The larger the ratio, the steeper is the latter function near absolute threshold. One would expect that the steeper the $\log \left(T_{\text {eff }}\right)$ vs. SPL function near absolute threshold, the steeper should be the psychometric function relating correct detection to SPL and, consequently, the smaller should be the variability of threshold estimates. Figure 10 shows that for each cat mean SDs are indeed considerably smaller after than before hearing loss. The ratios of $P_{\text {ineff }}$ to $T_{1}$ are also larger after than before hearing loss by about 3 orders of magnitude for the grand mean (see above and Fig. 5). The average decrease of the mean SDs (large symbols in Fig. 10) is about $3.4 \mathrm{~dB}$, from $6.3 \mathrm{~dB}$ before to $2.9 \mathrm{~dB}$ after hearing loss. This number compares reasonably well with the $5.6 \mathrm{~dB}$ expected from the change in slope $s$ of the $\log \left(T_{\text {eff }}\right)$ vs. SPL functions near absolute thresholds when $P_{\text {ineff }}$ increases from 5 $\mathrm{nPa}$ to $77 \mu \mathrm{Pa}$ (values for grand mean data before and after hearing loss). Using Eq. (3), slopes were calculated at the SPLs which correspond to the mean amplitude of each stimulus at its absolute threshold. The ratios of the two slopes for each stimulus after to before hearing loss were expressed in $\mathrm{dB}$ and then averaged across all stimuli. The $5.6 \mathrm{~dB}$ and $3.4 \mathrm{~dB}$ changes in SDs translate into slopes of the $\log \left(T_{\text {eff }}\right)$ vs. SPL function at absolute threshold and for $P_{\text {ineff }}=77$ $\mu \mathrm{Pa}$ of 1.9 and 1.5 , respectively (those for $5 \mathrm{nPa}$ are essentially 1).

Thus, these data provide strong support for the notion that $\log \left(T_{\text {eff }}\right)$ vs. SPL functions near absolute thresholds in these cats are steeper after compared 

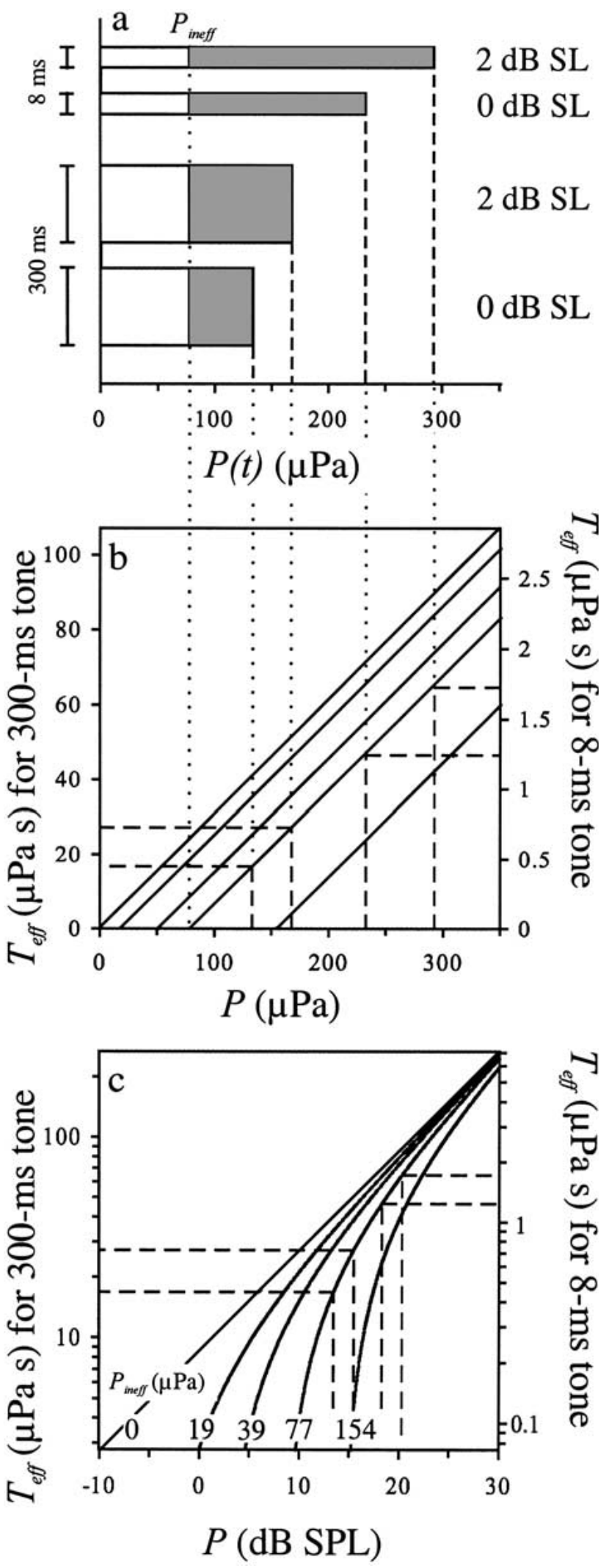

with before hearing loss and that they are steeper than 1 after hearing loss. The slopes predicted by Figure $9 \mathrm{c}$ constitute the upper limits that are possible
FIG. 9. Consequences of an elevated baseline for effective stimulus growth above threshold. a. Schematic stimuli of rectangular temporal envelope with durations of 300 and $8 \mathrm{~ms}$. The two stimuli labeled $0 \mathrm{~dB}$ SL have amplitudes that correspond very closely to those extrapolated from the grand mean thresholds of the cats after hearing loss. The value of $P_{\text {ineff }}=77 \mu \mathrm{Pa}$ also equals that obtained from these data. Each stimulus consists of an effective portion, $T_{\text {eff, }}$ specified as the temporal integral of $P_{\text {eff }}(t)$ (gray areas), and an ineffective portion, the temporal integral of $P_{\text {ineff }}$ (white areas). $P_{\text {ineff }}$ does not change with level. $\mathbf{b}$. Plot of $T_{\text {eff }}$ against the maximum amplitude of $P(t), P$, for stimuli of rectangular temporal envelope. The diagonal is for $P_{\text {ineff }}=0$ and the other parallel lines are for values of $P_{\text {ineff }}=19,39,77$, and $154 \mu \mathrm{Pa}$ (from left to right, cf. c). Note the linear growth of $T_{\text {eff }}$ once $P(t)>P_{\text {ineff. }}$. Dashed lines emanating from the function for $P_{\text {ineff }}=77 \mu \mathrm{Pa}$ identify the values of $P$ (abscissa; cf. a) and of $T_{\text {eff }}$ for stimuli of 0 and $2 \mathrm{~dB} \mathrm{SL}$, with $P_{\text {ineff }}=77 \mu \mathrm{Pa}$ and with durations of 300 (left ordinate) and $8 \mathrm{~ms}$ (right ordinate). c. Same data as in $\mathbf{b}$ plotted in double-log coordinates (values of $P$ expressed in $\mathrm{dB} \mathrm{SPL}$ ).

with an elevated baseline. These limits are obtained under the assumption of a step-like transition of the effectiveness of the pressure from 0 to 1 at $P_{\text {ineff. The }}$ smaller change in slopes obtained from the above analyses of SDs may thus be attributable to non-steplike, more gradual transitions. Figure 11a shows a few possible transitions, including the step function (with $\left.P_{\text {ineff }}=77 \mu \mathrm{Pa}\right)$, and Figure $11 \mathrm{~b}$ shows the resulting $\log \left(T_{\text {eff }}\right)$ vs. SPL functions, as in Figure 9c. Figure 11 illustrates that, compared with a step function (function 2), smooth transitions of effectiveness (functions 4 and 6) produce shallower growths of $\log \left(T_{\text {eff }}\right)$ with $\log (P)$ at very low levels.

The curves for $P_{\text {ineff }}>0$ in Figure $9 \mathrm{c}$ also bear some resemblance to recruitment curves or loudness balance functions obtained from loudness matches, at least to a first approximation. The former are plots of loudness along the ordinate against stimulus level, and the latter are plots of the levels of a stimulus presented to a normal ear along the ordinate versus the levels of that stimulus required to produce equal loudnesses when presented to an impaired ear (e.g., Hellman 1997; Buus and Florentine 2002; Moore 2004). A direct comparison of such plots with Figure $9 \mathrm{c}$ is problematic since the relationship between $T_{\text {eff }}$ and loudness is unknown. Still, let us assume that loudness is some monotonically increasing function of $T_{\text {eff }}$. Then the steep growth of the functions for $P_{\text {ineff }}>0$ just above $P_{\text {ineff }}$ seems at variance with findings that the growth of loudness with level in human subjects can be similar in normal and impaired ears for levels within a few $\mathrm{dB}$ (up to $4-10 \mathrm{~dB}$, depending on subject) of absolute threshold (Buus and Florentine 2002; Moore 2004). How can these findings be reconciled with our model of an elevated baseline? Again, the actual transitions from $P_{\text {ineff }}$ to $P_{\text {eff }}$ may be more gradual. In addition, it is conceivable that the effectiveness of the pressure below $P_{\text {ineff }}$ may be re- 


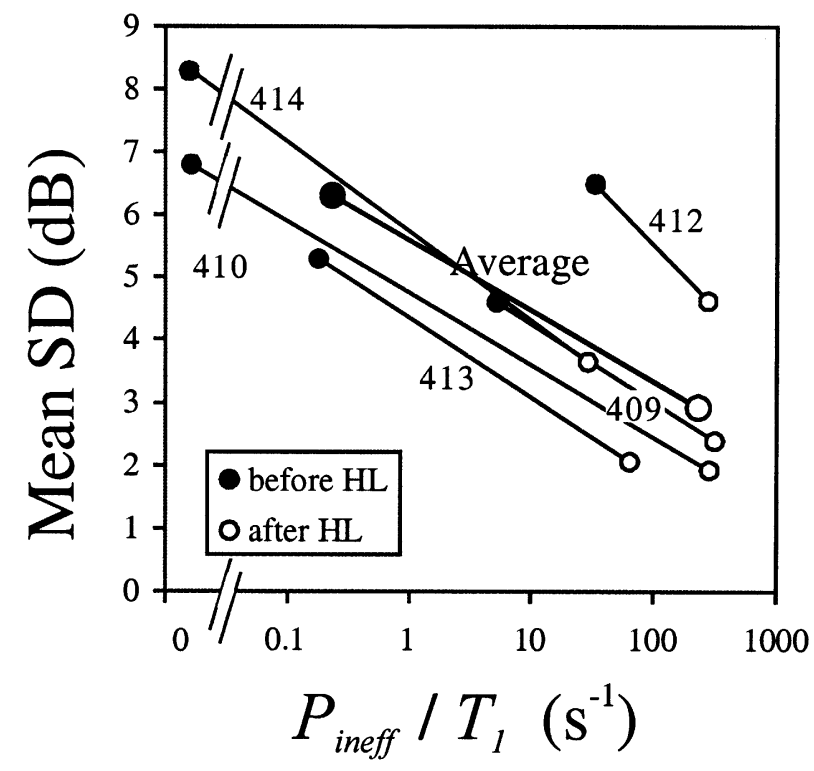

FIG. 10. Experimental evidence for the prediction of steeper $\log \left(T_{\text {eff }}\right)$ vs. SPL functions after compared to before hearing loss (cf. Fig. 9c). For each cat, the SDs of the 12 accepted thresholds for each stimulus were averaged across all 10 stimuli to yield a mean SD (in $\mathrm{dB}$ ) before (filled symbols) and after (open symbols) hearing loss. The larger symbols represent the average of these mean SDs across the five cats. Mean SD is plotted against the ratio of $P_{\text {ineff }}$ to $T_{1}$ (in s ${ }^{-1}$ ), that parameter which is proportional to the steepness of the $\log \left(T_{\text {eff }}\right)$ vs. SPL functions. The decrease of mean SD parallels the increase in the ratio of $P_{\text {ineff }}$ to $T_{1}$, with similar slopes in different cats. For cats 410 and 414 , the estimates of $P_{\text {ineff }}$ were zero, and consequently $P_{\text {ineff }} / T_{1}$ is zero (note interrupted abscissa and lines connecting data points). Nevertheless, the distance of these symbols from the others is as if $P_{\text {ineff }}$ in these cases had been about $1 \mathrm{nPa}$, the limit of accuracy of the Solver used to fit the data. The position along the abscissa of the symbols representing the average corresponds to that of the grand mean data. The decrease in mean SD after hearing loss is expected if the $\log \left(T_{\text {eff }}\right)$ vs. SPL functions near absolute thresholds are steeper, since then the psychometric functions relating correct detection to SPL should be steeper and the variability of threshold estimates smaller.

duced not quite to zero, particularly if the hearing loss was not caused by a single massive traumatic event but by a more gradual process. Consequently, some small residual effectiveness may remain or may redevelop over time after some traumatic event. Figure 11 illustrates that a residual effectiveness produces a slope of 1 of $\log \left(T_{\text {eff }}\right)$ vs. $\log (P)$ functions at very low levels (functions 3,5, and 7). Thus, the near-normal growth of loudness observed in some listeners with sensorineural hearing losses at very low levels and the more rapid growth of loudness level above those levels are not inconsistent with an elevated baseline. This, of course, does not exclude the possibility that recruitment at higher SPLs in such listeners may be dominated by loss of the compressive nonlinearity of the $\mathrm{BM}$ response (for review see, e.g., Moore 1996).

Figure 9b,c also identifies, by dashed lines originating from the function for $P_{\text {ineff }}=77 \mu \mathrm{Pa}$, the val- ues of $P$ and of $T_{\text {eff }}$ for stimuli of 0 and $2 \mathrm{~dB}$ SL and of 300- (left ordinate) and 8-ms duration (right ordinate). It is evident from Figure $9 \mathrm{c}$ that the relative increment in $T_{\text {eff }}$ with the 2-dB increment in pressure is larger for the long tone than for the short tone (the function is steeper around the values for the long tones). In other words, to achieve the same relative increment in $T_{\text {eff }}$ (same distances between horizontal lines in Fig. 9c) for the short and the long tone, a larger increment in level is required for the short than for the long tone. The same would hold for other values of $P_{\text {ineff, }}$ as long as they exceed zero. Again, assume that some suprathreshold response measure were a function of $T_{\text {eff. }}$ Then, our model predicts a steeper growth of that response with a given increment in stimulus level for long than for short tones, when $P_{\text {ineff }}>0$. Viewed from the other perspective, to obtain a given suprathreshold response, a larger sensation level is needed for a short than for a long tone, when $P_{\text {ineff }}>0$. This prediction fits in nicely with findings of Stelmachowicz and Seewald (1977). These authors measured absolute thresholds and stapedial reflex thresholds (requiring higher SPLs than absolute thresholds) to tones of 25and 250-ms durations, in normal-hearing subjects and in subjects with sensorineural hearing losses. Their results (for $1-\mathrm{kHz}$ tones) are illustrated in Figure 12. Their data show that in normal-hearing subjects the amount of temporal integration, i.e., the difference in threshold SPLs between the short and the long tone, is about the same for absolute and for stapedial reflex thresholds. Thus, to reach the stapedial reflex threshold required about the same SL for the short and the long tone. In the subjects with sensorineural hearing losses, however, the amount of temporal integration was smaller, by about $5 \mathrm{~dB}$, for absolute than for stapedial reflex thresholds. Thus, to reach the stapedial reflex threshold a higher SL was required for the short than for the long tone, just as predicted by our model. For the absolute thresholds, the amount of temporal integration was smaller than in normal-hearing subjects, but for stapedial reflex thresholds, the amounts of temporal integration were about the same in normal-hearing and in hearingimpaired subjects.

"Integration" and reaction times. Our EBTS model also predicts that "integration" times for stimuli of the same nominal SL should be shorter when $P_{\text {ineff }}>$ 0 , i.e., with sensorineural hearing loss, compared to when $P_{\text {ineff }}=0$, i.e., with normal hearing. For simplicity, let us again consider stimuli of rectangular temporal envelope. For a stimulus of a given duration $t_{\mathrm{s}}$, threshold is reached, on average, when $P(t)=P_{\mathrm{T}}$. Viewed the other way, and per definition, given $P_{\mathrm{T}}$ the observer has to "integrate," again on average, over a time $t_{i}\left(P_{\mathrm{T}}\right)=t_{\mathrm{s}}$ before the stimulus is detected 

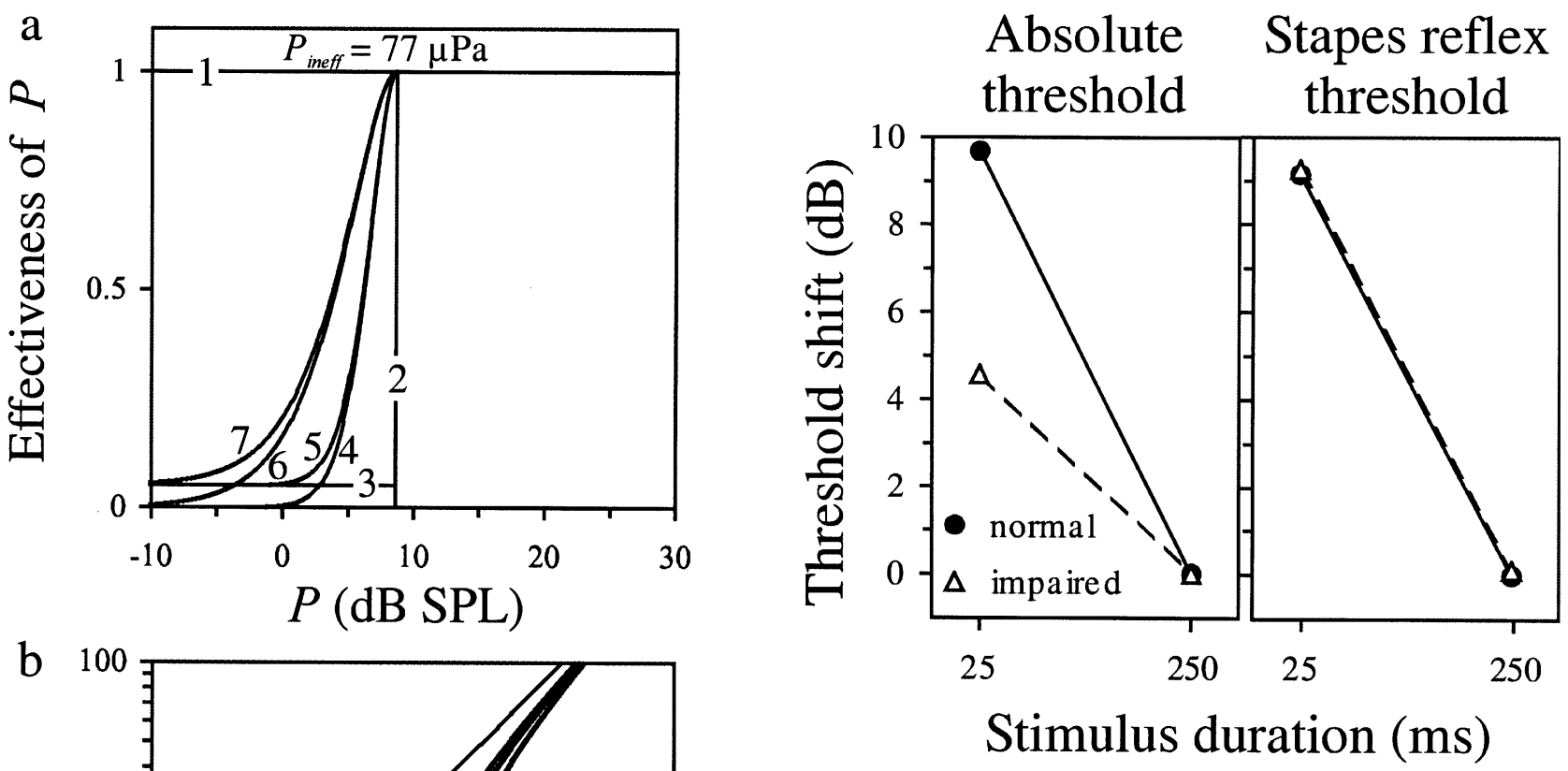

FIG. 12. Mean shift (in $\mathrm{dB}$ ) in threshold for a 25-ms relative to a 250-ms duration tone of $1 \mathrm{kHz}$ for absolute threshold and stapedial reflex threshold for 20 normal-hearing subjects and 20 subjects with sensorineural hearing losses. Modified from Figure 2 of Stelmachowicz and Seewald (1977).

$P_{\text {ineff }}=0$, the $1-\mathrm{dB}$ increment in amplitude is equivalent to a factor of 1.09 , and Eq. (1) predicts that $t_{\mathrm{i}}$ $\left(1.09 P_{\mathrm{T}}\right)=1.09^{1 /(m-1)} t_{\mathrm{s}}$. For $m=0.69$, the value derived from the grand mean data of Solecki and Gerken $(1990)$, we obtain $t_{i}\left(1.09 P_{\mathrm{T}}\right)=0.76 t_{\mathrm{s}}$ (Fig. 13a, top right). In subjects with sensorineural hearing loss, where $P_{\text {ineff }}>0$, the situation at absolute threshold is similar to normal-hearing subjects, in so far as the observer has to "integrate" over a time $t_{i}\left(P_{\mathrm{T}}\right)=t_{\mathrm{s}}$ before the stimulus is detected, although $P_{\mathrm{T}}=P_{\text {eff }}+$ $P_{\text {ineff }}$ (Fig. 13a, bottom left). But now if the amplitude is raised by $1 \mathrm{~dB}, P_{\text {eff }}$ is raised by more than a factor of 1.09 because $P_{\text {ineff }}$ remains the same (in Fig. 13a by a factor of 1.29 , equivalent to $2.2 \mathrm{~dB}$ ). Hence, the "integration" time reduces to less than $0.76 t_{\mathrm{s}}$, the time for $P_{\text {ineff }}=0$ (Fig. 13a, bottom right). The absolute difference in "integration" times between normal-hearing subjects, where $P_{\text {ineff }}=0$, and hearing-impaired subjects, where $P_{\text {ineff }}>0$, depends, with other parameters constant, on the magnitude of $P_{\text {ineff }}$ (the larger $P_{\text {ineff, }}$, the larger the difference), on the reduction in sensitivity (the larger the reduction, the smaller the difference), on the stimulus duration (the longer $t_{\mathrm{s}}$, the larger the difference), and on the sensation level (once SL exceeds 1-2 dB, the difference decreases with increasing SL). The shape of the difference versus sensation level function also varies with $m$. If there are no differences in the average processing speed and on the motor side of the sensorymotor loop (of course, these parameters will be dis-

(Fig. 13a, left). Now, if the amplitude is raised, for example by $1 \mathrm{~dB}$ (as in Fig. 13a), the average "integration" time required to detect the stimulus shortens (Fig. 13a, right). With normal hearing, where 
“integration" time required to detect the stimuli, just as predicted by an elevated baseline in our model.

\section{DISCUSSION}

We have shown here that the changes in absolute thresholds to tones of different envelopes and durations consequent upon noise trauma can be well described as resulting from a reduction in sensitivity, or gain, and from an elevation of the baseline above which sound pressure becomes effective in driving the auditory system. The idea of an elevated baseline appears similar to that of a threshold intensity or that of an internal noise that needs to be exceeded before a stimulus can have an effect on the auditory system. Such ideas have been introduced to models of loudness growth (e.g., Zwislocki 1965; Zwicker and Feldtkeller 1967; see also Nielsen 1993; Humes and Jesteadt 1991; Hellmann 1997; Buus and Florentine 2002).

Our EBTS model fully accounts for the mismatch of thresholds, when specified in terms of integrated $P(t)$, to single- and multiple-burst stimuli of the same duration and for the steeper increase in such thresholds with duration after hearing loss (Figs. 2 and 4). In other words, once the elevated baseline is taken into account and thresholds are specified as the temporal integral of $P_{\text {eff }}(t)$, the remaining differences in thresholds before and after hearing loss can be captured by a simple gain change. Thus, an elevated baseline is a very attractive concept because it allows us to assume that those processes that underlie the specific slope of the trading relationship between amplitude and duration of threshold stimuli are unaltered, irrespective of which processes these may be (Moore 1996; Krishna 2002; Heil and Neubauer 2003). Our specific proposal is that thresholds are reached by summation of chance events where each of these events is thought to require the interaction of about four statistically independent, or nearly independent, subevents. We proposed that the events can be traced back to exocytotic events at the inner hair cell (IHC) to auditory-nerve fiber synapses, where spikes are first generated, and hypothesized that the subevents might be Ca-binding steps required for exocytosis (Heil and Neubauer 2003, $2004 \mathrm{a}, 2004 \mathrm{~b})$. This idea of a critical role of the presynapses of the IHC was derived from a reformulation of Eq. (1) with $m \cong 0.75$, the weighted average across all species, which yielded $1 /(1-m)=4$ such subevents, and the fact that exocytosis depends on about the 4th power of the intracellular Ca concentration in all synapses studied in detail (e.g., Beutner et al. 2001; Meinrenken et al. 2003). Thus, our finding here that $m$ is not significantly changed following hearing loss, when the elevated baseline is taken into account, supports our view that the Ca mechanism is also not changed.

Location of damages captured by altered baseline and gain

Thus, if the true slope of the temporal integration function is unaltered by the trauma, and if that slope reflects the nonlinear dependence of exocytosis on the Ca concentration in the IHC, then our model predicts that the damage, captured by $P_{\text {ineff }}$, must be peripheral to the processes governing exocytosis from the IHC. Conductive hearing losses, e.g., due to otosclerosis, do not seem to affect the slope of conventional temporal integration functions (MiskolczyFodor 1953; Harris et al. 1958; preliminary data by Wright 1968; but see Papsin and Abel 1988), although they may disguise alterations of the slope due to additional sensorineural defects (Harris et al. 1958; see also Fig. 6). This suggests as a likely candidate site the inner ear, including structures involved in mechanoelectrical coupling. For example, the contact between the tectorial membrane and the stereocilia of the outer hair cells (OHCs) or the tip links between stereocilia of IHCs or OHCs may be damaged, so that the chain of sound-evoked vibrations is interrupted when the amplitudes of the vibrations are small. Only amplitudes exceeding some limit, captured by $P_{\text {ineff, }}$ are transduced further along the chain. It is very likely that $P_{\text {ineff }}$ varies with frequency, since damage due to noise trauma, presbyacusis, ototoxic drugs, or diseases is not uniform along the cochlea. As stated in the Results, a small $P_{\text {ineff }}$ may also be present in subjects classified as normal-hearing. If in such subjects $P_{\text {ineff }}$ would also vary with frequency, then this could underlie the frequencydependent differences in the slopes of temporal integration functions sometimes reported (e.g., Watson and Gengel 1969; Florentine et al. 1988).

The damages that result in reduced sensitivity or gain after noise trauma are more difficult to locate because very peripheral mechanisms, such as those producing conductive hearing loss, as well as presumably central processes such as attention or expectations (Dai and Wright 1995), can affect the sensitivity, possibly also via efferent feedback (e.g., Puel et al. 1988; Ruel et al. 2001). Nevertheless, damage within the inner ear is most likely to contribute. Noise trauma can have a range of chronic effects, such as stereocilia damage, loss of OHCs and IHCs, complete loss of the organ of Corti, and many more, with differences in spatial distribution, time course, and dependence on noise level (and presumably exposure duration) (e.g., Saunders et al. 1985; Liberman 1987, 1990; Wang et al. 2002). 
Other models to account for altered slopes of temporal integration functions

Several ideas have been proposed to account for the reduced slope of the temporal integration function in listeners with sensorineural hearing losses. However, the more likely ones have not survived critical tests. For example, in listeners with steep sensorineural hearing losses, one might think that the reduced slope could result from the detection of spectral splatter to frequencies where thresholds are lower. However, that possibility has been ruled out (Florentine et al. 1988; Carlyon et al. 1990). Similarly, Florentine et al. (1988) thoroughly tested and rejected the idea that the reduced slope may be due to the higher SPLs of the stimuli needed to reach absolute threshold in hearing-impaired listeners, and they concluded that it reflects reduced temporal integration per se. Saunders et al. (1995), in following the idea that the integrator may be located centrally (e.g., Zwislocki 1960), have suggested that the neural integrator exhibits considerable plasticity since a normal slope of the temporal integration function can be restored in the chicken when damaged structures in the avian cochlea regenerate. However, there is no need to assume such plasticity if the integrator is located very peripherally, as suggested by us (Heil and Neubauer 2003).

Moore $(1991,1996)$ has suggested that the reduced slope of the temporal integration function in hearing-impaired listeners is due to a reduction or loss of the compressive nonlinearity of the basilar membrane (BM) (see also Oxenham et al. 1997; Moore and Oxenham 1998; Oxenham and Bacon 2003). The model assumes that, due to the loss of OHCs, the BM input-output (I/O) function becomes linear and that, as a consequence of the steeper increase in BM displacement or velocity with a given increment in stimulus level, the firing rate versus level functions of auditory-nerve fibers also become steeper. For the model to work, the proposed steepening of the firing rate versus level functions of auditorynerve fibers must occur also at very low levels, i.e., near those at which firing rate starts to increase. For this steepening to result from a reduction of compression, the normal $\mathrm{BM} \mathrm{I} / \mathrm{O}$ function must be compressively nonlinear, even at very low SPLs. All currently available measurements of BM mechanics, however, seem to agree that at characteristic frequency $(\mathrm{CF})$ the $\mathrm{BM}$ displacement and velocity grow linearly with pressure at low SPLs and are compressively nonlinear at higher SPLs (Sellick et al. 1982; Robles et al. 1986; Ruggero et al. 1997; Zinn et al. 2000; Overstreet et al. 2002; see also Yates et al. 1990; Oxenham et al. 1997; Buus and Florentine 2002). While these data stem from anesthetized animals and cochleae of physiological states that may differ to some extent from normal, they provide no evidence for compression at very low SPLs. Furthermore, authors who use techniques or models designed to derive the $\mathrm{BM} \mathrm{I} / \mathrm{O}$ function indirectly, for example, through frequency-dependent changes in the shape of firing rate versus level functions of auditory-nerve fibers (Sachs and Abbas 1974; Yates et al. 1990, 1992; Cooper and Yates 1994) or forward masking (Plack and O'Hanlon 2003), claim to see in their measures the reflections of a linear BM response at low levels. Only, one recent modeling study assumes compressive growth at very low SPLs (Eguiluz et al. 2000). Even if all current measurements of BM mechanics were compromised and the $\mathrm{BM} \mathrm{I} / \mathrm{O}$ function were in fact compressively nonlinear even at low SPLs, it is unclear whether the loss of this compression would lead to steeper firing rate versus level functions of auditory-nerve fibers, as proposed by Moore (1991, 1996). This is particularly doubtful for the most sensitive fibers which would be expected to mediate absolute thresholds. Yates et al. (1990) recorded rate versus level functions of auditory-nerve fibers in guinea pigs to tones at CF and at a "tail" frequency well below CF where the BM response is linear (e.g., Ruggero et al. 1997; Zinn et al. 2000). Yates et al. reported that for a given fiber the shapes of the functions at these different frequencies are essentially identical at low firing rates up to some rate beyond which they deviate. The firing rate at which the break occurs depends on the fiber's spontaneous firing rate and hence on its sensitivity. For fibers of high spontaneous rates, which are the most sensitive ones, firing rate versus level functions at $\mathrm{CF}$ and at tail frequencies are essentially identical in shape from the lowest to the highest firing rates. We made similar observations on cat auditory-nerve fibers (Heil, Brown, Irvine, unpublished). Also, Heinz and Young (2004) reported that the growth of discharge rates of auditory-nerve fibers to tones and at low levels (lowlevel slope) was not consistently steeper in cats with noise-induced hearing losses compared to normal cats. A more detailed analysis revealed that the lowlevel slopes of those fibers from hearing-impaired cats that were broadly tuned to frequency were slightly shallower. However, these fibers tended to be among those whose thresholds appeared most severely elevated due to the noise trauma and, thus, they are unlikely to be critical for mediating absolute thresholds.

Our model of an elevated baseline in combination with integration of the pressure envelope (i.e., $q=1$ ) (EBTS model) does not require altered compression of the BM at low levels nor altered shapes of rate versus level functions of auditory-nerve fibers at low firing rates to explain altered rates of temporal inte- 
gration for absolute thresholds in quiet in subjects with sensorineural hearing losses. Also, it provides a substantially better description of the data than an altered power-law compression (Fig. 7). We are not aware of any physiological or psychoacoustic observations that are at variance with it.

\section{ACKNOWLEDGMENTS}

We are most grateful to Janet M. Solecki and George M. Gerken for putting the cat threshold SPLs at our disposal. We also thank Michael Brosch, Ray Meddis, and three anonymous reviewers for helpful comments on earlier versions of the manuscript, and Henning Scheich for setting up a favorable framework. This study was supported by grants of the Deutsche Forschungsgemeinschaft to Peter Heil.

\section{REFERENCES}

Beutner D, Voets T, Neher E, Moser T. Calcium dependence of exocytosis and endocytosis at the cochlear inner hair cell afferent synapse. Neuron 29:681-690, 2001.

Brahe Pedersen C, Elberling C. Temporal integration of acoustic energy in patients with presbyacusis. Acta Otolaryngol. 75:3237, 1973.

Brahe Pedersen C, Salomon G. Temporal integration of acoustic energy. Acta Otolaryngol. 83:417-423, 1977.

Buser P, Imbert M. Audition. MIT Press, Cambridge, 1992.

Buus S, Florentine M. Growth of loudness in listeners with cochlear hearing losses: recruitment reconsidered. J. Assoc. Res. Otolaryngol. 3:120-139, 2002.

Buus S, Florentine M, Poulsen T. Temporal integration of loudness in listeners with hearing losses of primarily cochlear origin. J. Acoust. Soc. Am. 105:3464-3480, 1999.

Carlyon RP, Buus S, Florentine M. Temporal integration of trains of tone pulses by normal and cochlearly impaired listeners. J. Acoust. Soc. Am. 87:260-268, 1990.

Chung DY, Sмiтh F. Quiet and masked brief-tone audiometry in subjects with normal hearing and with noise-induced hearing loss. Scand. Audiol. 9:43-47, 1980.

Clark WW, Bohne BA. "Cochlear damage. Audiometric correlates". In: Collins MJ, Glattke TJ, Harker LA (eds), Sensorineural Hearing Loss, University of Iowa, Iowa City, pp 59-82, 1986.

Clock Eddins A, Peterson JR. Time-intensity trading in the late auditory evoked potential. J. Speech Lang. Hear Res. 42:516$525,1999$.

Clock Eddins A, Salvi RR, Saunders SS, Powers NL. Neural correlates of temporal integration in the cochlear nucleus of the chinchilla. Hear Res. 71:37-50, 1993.

Clock Eddins A, Salvi RR, Wang J, Powers NL. Threshold-duration functions of chinchilla auditory nerve fibers. Hear Res. 119:135141, 1998.

Cooper NP, Yates GK. Nonlinear input-output functions derived from the responses of guinea-pig cochlear nerve fibres: variations with characteristic frequency. Hear Res. 78:221-234, 1994.

Costalupes JA. Temporal integration of pure tones in the cat. Hear Res. 9:43-54, 1983.

DAI H, WrIGHT BA. Detecting signals of unexpected and uncertain durations. J. Acoust. Soc. Am. 98:708-896, 1995.
Dallos PJ, Johnson KR. Influence of rise-fall time upon short-tone threshold. J. Acoust. Soc. Am. 40:1160-1163, 1966.

Dau T, Püschel D, Kohlraush A. A quantitative model of the "effective", signal processing in the auditory system. I. Model structure. J. Acoust. Soc. Am. 99:3615-3622, 1996a.

Dau T, Püschel D, Kohlrausch A. A quantitative model of the "effective" signal processing in the auditory system. II. Simulations and measurements. J. Acoust. Soc. Am. 99:3623-3631, 1996b.

Davis RI, Ferraro JA. Temporal summation in normally and abnormally hearing chinchillas. J. Aud. Res. 23:281-292, 1983.

DEBoer, E. “Auditory time constants: a paradox?" In: Michelson, A (ed), Time Resolution in the Auditory System, Springer-Verlag, Heidelberg, pp 141-155, 1985.

Dempsey JJ, Maxon AB. Temporal integration functions in hearingimpaired children. Ear Hear 3:271-273, 1982.

DunIA R, NARINS PM. Temporal integration in an anuran auditory nerve. Hear Res. 39:287-297, 1989.

Eguiluz VM, Ospeck M, Choe Y, Hudspeth AJ, Magnasco MO. Essential nonlinearities in hearing. Phys. Rev. Lett. 84:5232$5235,2000$.

ELLIOTT LL. Tonal thresholds for short-duration stimuli as related to subject hearing level. J. Acoust. Soc. Am. 35:578-580, 1963.

FAY RR. "Structure and function in sound discrimination among vertebrates". In: Webster DB, FAY RR, Popper AN (eds), The Evolutionary Biology of Hearing, Springer-Verlag, New York, pp 229-263, 1992.

Feldtkeller R, Oetinger R. Die Hörbarkeitsgrenzen von Impulsen verschiedener Dauer. Acustica 6:489-493, 1956.

Florentine M, Fastl H, Buus S. Temporal integration in normal hearing, cochlear impairment, and impairment simulated by masking. J. Acoust. Soc. Am. 84:195-203, 1988.

Florentine M, Buus S, Rosenberg M. "Reaction-time data support the existence of softness imperception in cochlear hearing loss"'. In: Pressnitzer D, de Cheveigne A, mcAdams S, Collet L (eds), Auditory Signal Processing: Physiology, Psychoacoustics, and Models, Springer, New York, 2004.

GARNER WR. The effect of frequency spectrum on temporal integration of energy in the ear. J. Acoust. Soc. Am. 84:195-203, 1947.

Gengel RW, Watson CS. Temporal integration: I. Clinical implications of a laboratory study. II. Additional data from hearingimpaired subjects. J. Speech. Hear Disord. 36:213-224, 1971.

Gerken GM, Bhat VKH, Hutchison-Clutter M. Auditory temporal integration and the power function model. J. Acoust. Soc. Am. 88:767-778, 1990.

Gorga MP, Beauchaine KA, Reiland JK, Worthington DW, Javel E. The effects of stimulus duration on $\mathrm{ABR}$ and behavioral thresholds. J. Acoust. Soc. Am. 76:616-619, 1984.

Green DM “Temporal factors in psychoacoustics”. In: Michelsen, A (ed), Time resolution in the auditory system. Springer-Verlag, Heidelberg, pp 122-140, 1985.

Hall JW, Fernandez MA. Temporal integration, frequency resolution, and off-frequency listening in normal-hearing and cochlea-impaired listeners. J. Acoust. Soc. Am. 74:1172-1177, 1983.

Hall JW, Wood EL. Stimulus duration and frequency discrimination for normal-hearing and hearing-impaired subjects. J. Speech Hear Res. 27:252-256, 1984.

Harris JD, Haines HL, Myers CK. Brief-tone audiometry. Temporal integration in the hypacusic. AMA Arch. Otolaryngol. (Chicago) 67:699-713, 1958.

Heil P, Neubauer H. Temporal integration of sound pressure determines thresholds of auditory-nerve fibers. J. Neurosci. 21:7404-7415, 2001. 
Heil P, Neubauer H. A unifying basis of auditory thresholds based on temporal summation. Proc. Natl. Acad. Sci. USA 100:61516156, 2003.

Heil P, Neubauer H. "Auditory thresholds re-visited". In: Pressnitzer D, de Cheveigne A, McAdams S, Collet L (ed), Auditory Signal Processing: Physiology, Psychoacoustics, and Models, Springer, New York, pp 454-470 2004a.

Heil P, Neubauer H. "Towards a unifying basis of auditory threesholds". In: König R, Heil P, Budinger E, Scheich H (ed), The Auditory Cortex A Synthesis of Human and Animal Research, Lawrence Erlbaum Associates, Oxford (2004b).

HeInz MG, Young ED. Response growth with sound level in auditory-nerve fibers after noise-induced hearing loss. J. Neurophysiol 91:784-795, 2004.

Hellman RP. "Growth of loudness in sensorineural impairment: experimental results and modeling implications". In: JESTEADT W (ed), Modeling sensorineural hearing loss, Lawrence Erlbaum Associates, Mahwah, NJ, pp 199-212 1997.

Henderson D. Temporal summation of acoustic signals by the chinchilla. J. Acoust. Soc. Am. 46:474-475, 1969.

Hick ML, BACON SP. Effects of aspirin on psychophysical measures of frequency selectivity, two-tone suppression, and growth of masking. J. Acoust. Soc. Am. 46:1436-1451, 1999.

Humes LE, Jesteadt W. Models of the effects of threshold on loudness growth and summation. J. Acoust. Soc. Am 90:19331943, 1991.

Kidd G Jr, Mason CR, Feth LL. Temporal integration of forward masking in listeners having sensorineural hearing loss. J. Acoust.Soc. Am. 75:937-944, 1984.

Kitzes LM, Gibson MM, Rose JE, Hind JE. Initial discharge latency and threshold considerations for some neurons in cochlear nuclear complex of the cat. J. Neurophysiol. 41:1165-1182, 1978.

KRISHNA BS. A unified mechanism for spontaneous-rate, first-spike timing in the auditory nerve. J. Comp. Neurosci. 13:71-91, 2002.

Liberman MC. Chronic ultrastructural changes in acoustic trauma: serial-section reconstruction of stereocilia and cuticular plates. Hear Res 26:65-88, 1987.

Liberman MC. Quantitative assessment of inner ear pathology following ototoxic drugs or acoustic trauma. Toxicol. Pathol. 18:138-148, 1990.

Martin FN, WofFord MJ. Temporal summation of brief tones in normal and cochlea-impaired ears. J. Aud. Res. 10:82-86, 1970.

Meinrenken CJ, Borst JGG, Sakmann B. The Hodgkin-Huxley-Katz Prize Lecture: Local routes revisited: the space and time dependence of the $\mathrm{Ca} 2+$ signal for phasic transmitter release at the rat calyx of Held. J. Physiol. 547:665-689, 2003.

MiskolCZY-Fodor F. Monaural loudness-balance test and determination of recruitment-degree with short sound-impulses. Acta. Otolaryngol. (Stockh.) 43:573-595, 1953.

MOORE BCJ. Characterization and simulation of impaired hearing: implications for hearing aid design. Ear Hear 12(Suppl):154161, 1991.

MoOre BCJ. Perceptual consequences of cochlear hearing loss and their implications for the design of hearing aids. Ear Hear 17:133-161, 1996

MoOre BCJ. Testing the concept of softness imperception: Loudness near threshold for hearing-impaired ears. J. Acoust. Soc. Am. 115:3103-3111, 2004.

Moore BCJ, Oxenham AJ. Psychoacoustic consequences of compression in the peripheral auditory system. Psychol. Rev. 105:108-124, 1998.

Morita T, Naito Y, Nagamine T, Fujiki N, Shibasaki H, Ito J. Enhanced activation of auditory cortex in patients with inner-ear hearing impairment: a magnetoencephalographic study. Clin. Neurophysiol. 114:851-859, 2003.
Nielsen RB. An auditory model with hearing loss. The Acoustics Laboratory. Technical University of Denmark, Report No. 52, 1993.

O'Connor KN, Barruel P, Hajalilou R, Sutter ML. Auditory temporal integration in the rhesus macaque (Macaco mulatta). J. Acoust. Soc. Am 106:954-965, 1999.

Overstreet EH 3rd, Temchin AN, Ruggero MA. Basilar membrane vibrations near the round window of the gerbil cochlea. J. Assoc. Res. Otolaryngol. 3:351-361, 2002.

Oxenham AJ, BACON SP. Cochlear compression: perceptual measures and implications for normal and impaired hearing. Ear Hear 24:352-366, 2003.

Oxenham AJ, Moore BCJ, Vickers DA. Short-term temporal integration: evidence for the influence of peripheral compression. I Acoust Soc Am 101:3676-3687, 1997.

PAPSIN BC, ABEL SM. Temporal summation in hearing-impaired listeners. Otolaryngol. 17:93-100, 1988.

Penner MJ. A power law transformation resulting in a class of shortterm integrators that produce time-intensity trades for noise bursts. J. Acoust. Soc. Am. 63:195-201, 1978.

Plack CJ, O'Hanlon CG. Forward masking additivity and auditory compression at low and high frequencies. J. Assoc. Res. Otolaryngol. 4:405-415, 2003.

Plomp R, Bouman MA. Relation between hearing and duration for tone pulses. J. Acoust. Soc. Am. 31:794-758, 1959.

Puel JL, Bonfils P, Pujol R. Selective attention modifies the active micromechanical properties of the cochlea. Brain Res. 447:380 383, 1988.

Quaranta A, Sallustio V, Scaringi A. Cochlear function and speech recognition in the elderly. Audiology 40:301-307, 2001.

Robles L, Ruggero MA, Rich NC. Basilar membrane mechanics at the base of the chinchilla cochlea. I. Input-output functions, tuning curves, and phase responses. J. Acoust. Soc. Am. 80:1364-1374, 1986.

Ruel J, Nouvian R, Gervais d’Aldin C, Pujol R, Eybalin M, Puel JL. Dopamine inhibition of auditory nerve activity in the adult mammalian cochlea. Eur. L. Neurosci. 14:977-986, 2001.

Ruggero MA, Rich NC, Recio A, Narayan SS, Robles L. Basilarmembrane responses to tones at the base of the chinchilla cochlea. J. Acoust. Soc. Am. 101:2151-2163, 1997.

SACHs MB, ABbas PJ. Rate versus level functions for auditory-nerve fibres in cats: tone burst stimuli. J. Acoust. Soc. Am. 56:1835$1847,1974$.

SAunders JC, DeAr SP, Schneider ME. The anatomical consequences of acoustic injury: a review and tutorial. J. Acoust. Soc. Am. 78:833-860, 1985.

Saunders SS, SAlvi RJ, Miller KM. Recovery of thresholds and temporal integration in adult chickens after high-level $525-\mathrm{Hz}$ pure-tone exposure. J. Acoust. Soc. Am. 97:1150-1164, 1995.

Schmidt S, Thaller J. Temporal auditory summation in the echolocating bat, Tadarida brasiliensis. Hear Res. 77:125-134, 1994.

Sellickk PM, Patuzzi RB, Johnstone BM. Measurements of basilar membrane motion in the guinea pig using the Mossbauer technique. J. Acoust. Soc. Am. 72:131-141, 1982.

Solecki JM, Gerken GM. Auditory temporal integration in the normal-hearing, hearing-impaired cat. J. Acoust. Soc. Am. 88:779-785, 1990.

Stelmachowicz PG, Seewald RC. Threshold and suprathreshold temporal integration function in normal and cochlear-impaired subjects. Audiology 16:94-101, 1977.

Viemeister NF, WAKEFIELD GH. Temporal integration and multiple looks. J. Acoust. Soc. Am. 90:858-865, 1991.

Viemeister NF, Shivapuja BG, Recio A. "Physiological correlates of temporal integration”. In: Cazals Y, Horner K, Demany L (ed), Auditory Physiology and Perception, Pergamon Press, New York, pp 323-330 1992. 
WALl LG, Stephenson M. Temporal integration and presbycusis. J. Aud. Res. 22:210-215, 1982.

Wang Y, Hirose K, Liberman MC. Dynamics of noise-induced cellular injury and repair in the mouse cochlea. J. Assoc. Res. Otolaryngol. 3:2482-2468, 2002.

Watson CS, Gengel RW. Signal duration and signal frequency in relation to auditory sensitivity. J. Acoust. Soc. Am. 46:989-997, 1969.

WRIGHT HN. The effect of sensori-neural hearing loss on threshold-duration functions. J. Speech. Hear Res. 11:842-852, 1968.

YATES GK, WINTER IM, RoBERTSON D. Basilar membrane nonlinearity determines auditory nerve rate-intensity functions and cochlear dynamic range. Hear Res. 45:203-220, 1990.
Yates GK, Johnstone BM, Patuzzi RB, Robertson D. Mechanical preprocessing in the mammalian cochlea. Trends Neurosci. 15:57-61, 1992.

Zinn C, Maier H, Zenner H, Gummer AW. Evidence for active, nonlinear, negative feedback in the vibration response of the apical region of the in-vivo guinea-pig cochlea. Hear Res. 142:159-183, 2000.

Zwicker E, Feldtkeller R. Das Ohr als Nachrichtenempfnger. Hirzel, Stuttgart, 1967.

Zwislocki JJ. Theory of temporal auditory summation. J. Acoust. Soc. Am. 32:1046-1060, 1960.

ZwISLOCKI JJ. "Analysis of some auditory characteristics". In: LuCE RD, Bush RR, Galanter E (ed), Handbook of Mathematical Psychology, Wiley, New York, pp 1-97 1965. 